\title{
Nonadiabatic breakdown and pairing in high- $T_{c}$ compounds
}

\author{
L. Pietronero and E. Cappelluti \\ Dipartimento di Fisica, Università «La Sapienza», P. le A. Moro 2, 00185 Rome, Italy \\ INFM-CNR, SMC-Istituto dei Sistemi Complessi, CNR, v. dei Taurini 19, 00185 Rome, Italy \\ E-mail: Emmanuele.Cappelluti@roma1.infn.it
}

Received August 25, 2005

\begin{abstract}
The electron-phonon interaction plays a fundamental role on the superconducting and normal state properties of all the high- $T_{C}$ materials, from cuprates to fullerenes. Another common element of these compounds is in addition the extremely small Fermi energy $E_{F}$, which is comparable with the range $\omega_{\mathrm{ph}}$ of the phonon frequencies. In the situation the adiabatic principle $\omega_{\mathrm{ph}} / E_{F}<<1$, on which the standard theory of the electron-phonon interaction and of the superconductivity relies, breaks down. In this contribution we discuss the physical consequences of the breakdown of the adiabatic assumption, with special interest on the superconducting properties. We review the microscopic derivation of the nonadiabatic theory of the electron-phonon coupling which explicitly takes into account higher order electron-phonon scattering not included in the conventional picture. Within this context we discuss also the role of the repulsive electron-electron correlation and the specific phenomenology of cuprates and fullerides.
\end{abstract}

PACS: 74.10.+v, 63.20.Kr

Keywords: high- $T_{c}$ superconductivity, electron-phonon interaction, fullerenes.

\section{Introduction}

For many years the concept of superconductivity has been strictly associated with the electron-phonon interaction. One of the fingerprints of the phonon-based Migdal-Eliashberg (ME) theory in conventional low-temperature superconductors has been in fact the prediction and observation of many peculiar features which are a direct evidence of a phonon mediated superconductivity, for instance the isotope effect $\alpha_{T_{c}}$ on the critical temperature, the extraction of the electron-phonon (el-ph) coupling function $\alpha^{2} F(\omega)$ from tunneling experiments, phonon anomalies occurring at temperature $T=T_{c}$, etc. [1]. The belief that superconductivity was intimately related to an electron-phonon pairing was so strong that a semi-empirical upper limit for the critical temperature $T_{c}^{\max } \simeq 20-25 \mathrm{~K}$ was thought to be valid before the occurrence of lattice instabilities [2,3], in agreement with the maximum $T_{c} \simeq 23 \mathrm{~K}$ achieved in $\mathrm{Nb}_{3} \mathrm{Sn}$.
This phonon-based scenario was shaken in 1986 by the discovery of high- $T_{c}$ superconductivity in copper oxides [4] with $T_{c}$ 's up to $140 \mathrm{~K}$, well above the empirical limit $T_{c}^{\max } \sim 20-25 \mathrm{~K}$. In addition, the isotope effect on the critical temperature at the optimal doping $\delta_{\text {opt }}$ in cuprates was found to be unconventionally small $\alpha_{T_{C}} \lesssim 0.1$ [5], suggesting a nonphonon mediated mechanism. Following this perspective a large amount of work has been devoted in the two last decades to the study of purely electronic models to explain the high- $T_{c}$ superconductivity.

In the recent years however, the evidence of an important role of the electron-phonon interaction on many properties of the normal and superconducting state has been increasing. On one hand, the small value of $\alpha_{T_{C}}$ turned out to be a peculiarity of the optimal doping, whereas in the underdoped region $\alpha_{T_{C}}$ could be significantly larger, even higher than the BCS limit $\alpha_{T_{C}}=0.5[6,7]$. On the other hand a remarkable isotope effect on the zero temperature London penetration depth $\lambda_{L}(0)$ has been observed both in the nearly optimal and in the underdoped regime 
[8-10]. The finite isotope shift on $\lambda_{L}(0)$ has been related to an isotope effect on the effective electron mass $m^{*}$ through the relation $\lambda_{L}(0) \propto \sqrt{n_{s} / m^{*}}$, where $n_{s}$ is the superfluid density. The observation of a finite isotope effect on $\lambda_{L}(0)$ or on $m^{*}$ is highly puzzling since these quantities are expected to show strictly zero isotope effect in the conventional electron-phonon framework. The report of a finite isotope effect on $m^{*}$ can be thus regarded not only as an indication of an important role of the electron-phonon coupling, but also as an evidence of the unconventional nature of the el-ph interaction [11-15]. Further support to a significant electron-phonon coupling in cuprates comes from angle-resolved photoemission spectroscopy (ARPES). The kink of the electronic dispersion observed by these measurements was indeed claimed to be of phononic origin, since it shows a negligible dependence on doping, on temperature and on the angle direction along the Fermi surface [16]. Moreover an isotope shift, not only of the kink itself, but also of the high energy electronic dispersion, points out once more a dominant but still not well understood role of the electron-phonon interaction [17].

Motivated by this experimental scenario, there is nowadays a revamping interest about the unconventional role of the electron-phonon interaction in cuprates and its relation with superconductivity. The observation of high- $T_{C}$ superconductivity with critical temperatures up to $T_{c}^{\max } \sim 40 \mathrm{~K}$ in fullerenes [18-21] and in the recently discovered $\mathrm{MgB}_{2}$ [22], where the phononic origin of the superconducting pairing is widely accepted, points out that a phonon-based mechanisms can be actually a route for high- $T_{C}$ superconductivity, and it suggests a common mechanism for all these compounds.

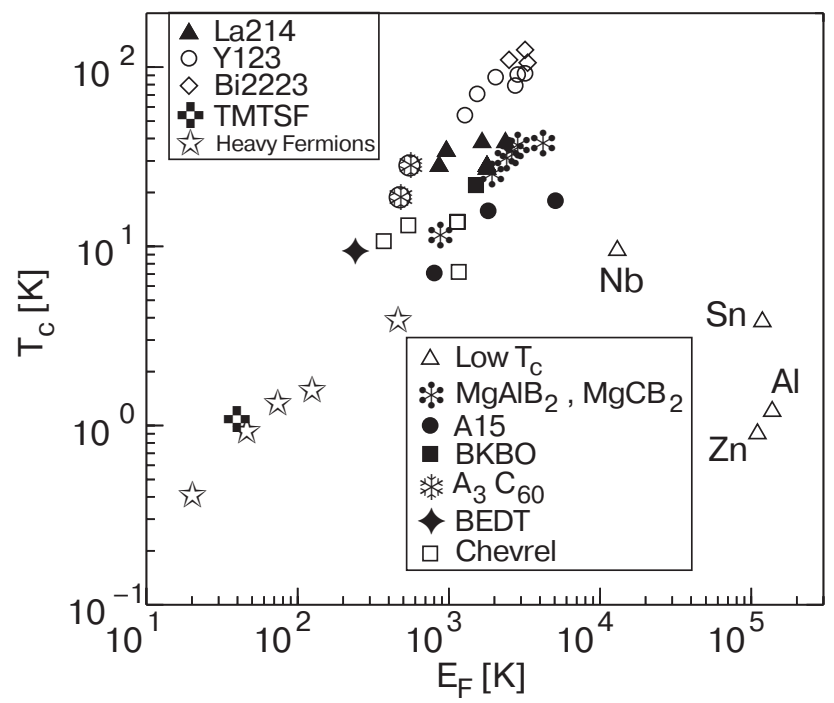

Fig. 1. Re-elaboration of $T_{C}$ vs. $E_{F}$ plot after Refs. 23, 24 including magnesium diboride alloys.
An useful insight on this issue is provided by the so called Uemura's plot $[23,24]$ (Fig. 1) which shows that high- $T_{c}$ and exotic superconductors (cuprates, fullerenes, but also heavy fermions, perovskites and Chevrel phases) are all characterized by a small density of charge carriers $n$, which is usually parametrized in terms of the Fermi energy $E_{F}$. This feature suggests that a positive role can be played by the small carrier density within an unique unconventional framework relevant for all these superconductors. This hypothesis could look quite puzzling because in conventional systems a low charge carrier density is usually considered detrimental for superconductivity since it decreases the number of metallic charges available for the Cooper pairing and it decreases the dynamical screening of the electron-electron Coulomb repulsion.

In the following we shall identify in the small Fermi energy $E_{F}$ induced by low values of $n$ the missing link between small carrier density and high- $T_{C}$ superconductivity. More precisely we shall see that when the electronic Fermi energy $E_{F}$ is small enough to be comparable with the characteristic energy of a generic boson mediator $\left(\omega_{\mathrm{ph}}\right.$ for the phononic case) one of the basic assumption of the conventional Migdal-Eliashberg theory, the adiabatic principle ${ }^{(1} \mathrm{ph} \ll E_{F}$, breaks down [25]. This situation calls for a generalization of the ME theory to explicitly include nonadiabatic effects in the same spirit as self-energy renormalization is taken into account in the ME theory itself with respect to the BCS one [26].

The review is structured as the following: in Sec. 2 we introduce the adiabatic problem and we discuss the implications arising from the failure of the adiabatic assumption in terms of the quantum field theory. In Sec. 3 we present the generalized theory of superconductivity in the nonadiabatic regime which takes into account the onset of nonadiabatic channels of interaction, while the relevance of the nonadiabatic scattering and the peculiar features of specific high- $T_{C}$ materials, as fullerenes and cuprates, will be discussed in Sec. 4 within the context of the nonadiabatic electron-phonon theory.

\section{Breakdown of Migdal's theorem}

\subsection{The nonadiabatic hypothesis}

One of the most popular principles in solid state physics is the adiabatic assumption which is on the basis of Born-Oppenheimer approximation [27]. Fundamental element of this approximation in metallic systems is the observation that the electron energy scale, parametrized by the Fermi energy $E_{F}$, is some orders of magnitude (typically $10^{3}-10^{4}$ ) larger than the lattice dynamics energy ruled by the phonon fre- 
quencies $\omega \mathrm{ph}$. This situation leads to the decoupling of the electron and lattice dynamics providing a suitable approximation scheme where the complex many-body problem becomes now affordable.

A powerful theoretical tool to deal with interacting electron systems is the quantum field theory which can be conveniently cast in terms of Feynman's diagrams $[28,29]$. The diagrammatic Feynman's approach results particularly useful when nonperturbative schemes are needed, as in the case of superconductivity, and in identifying classes of diagrams associated with a particular physical property. Feynman's theory provides indeed an elegant way to generalize the BCS theory in the strong coupling Migdal-Eliashberg regime.

From a generic point of view all the physical properties of an interacting electron system could be computed from the knowledge of the electron Green's function $G$ or, in an equivalent way, of the electron self-energy $\Sigma$, which is related to $G$ through the Dyson's equation [28]:

$$
G(\mathbf{k}, \omega)=[\omega-\varepsilon(\mathbf{k})-\Sigma(\mathbf{k}, \omega)]^{-1} .
$$

The self-energy itself can be in its turn expressed as a functional of the electron Green's function $G$, of the effective electron-electron potential $V$, and of the so called vertex function $\Gamma: \Gamma=\Gamma[G, V, \Gamma]$. In the electron-phonon case here considered, or for any electron-electron interaction mediated by a generic boson, the potential $V$ can be directly related to the phonon (boson) propagator $D$, so that

$$
\Sigma=\Sigma[G, D, \Gamma] .
$$

In the normal state Eq. (2) has the particular simple form:

$$
\Sigma(k)=-\int d k^{\prime} g\left(k-k^{\prime}\right) D\left(k-k^{\prime}\right) G\left(k^{\prime}\right) \Gamma\left(k^{\prime}, k^{\prime}-k\right),
$$

where the indexes $k$ and $k^{\prime}$ comprehend both frequencies and momenta and $g$ is the electron-phonon matrix element. A diagrammatic expression of Eq. (3) is shown in Fig. 2, where the solid line represents the electron propagator $G$, the wavy line the phonon

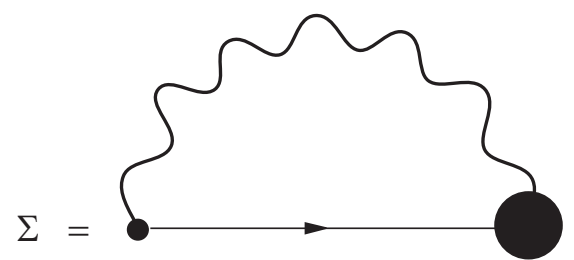

Fig. 2. Diagrammatic expression of the electron-phonon self-energy. The solid line represents the electron Green's function, the wary line the phonon propagator and the filled circles the electron-phonon vertex function.
Green's function $D$, and the filled circles the electron-phonon vertex function $\Gamma$. The complex manybody nature of the problem is thus hidden in the unknown quantity $\Gamma$ which in principle does not have an analytical expression. It is often useful to split the total vertex function into a zero-order constant term plus a vertex correction function denoted as $P$ :

$$
\Gamma\left(k^{\prime}, k^{\prime}-k\right)=g\left(k-k^{\prime}\right)\left[1+P\left(k^{\prime}, k^{\prime}-k\right)\right],
$$

where $P$ contains all the higher order interaction processes and it is constituted by an infinite set of diagrams.

The evaluation of the electronic self-energy, as expressed in Eq. (3), still constitutes a formidable task which cannot be analytically performed. A huge simplification to this aim was provided in the late 50ies by the so-called Migdal's theorem [25] which showed that, in the (adiabatic) limit $\omega_{\mathrm{ph}} / E_{F} \rightarrow 0$, the vertex correction function $P$ scales as

$$
P \propto \lambda \frac{{ }^{\omega} \mathrm{ph}}{E_{F}} .
$$

In common metals, as above discussed, we have $\omega_{\mathrm{ph}} / E_{F} \sim 10^{-3}-10^{-4}$. The total vertex function can be thus safely replaced by its lowest order (constant) term, $\Gamma \sim 1$ signalizing that the electron-phonon vertex function is not renormalized by strong-coupling effects in the adiabatic regime. In this framework the normal state self-energy simply reads thus:

$$
\Sigma(k)=-\int d k^{\prime} g^{2}\left(k-k^{\prime}\right) D\left(k-k^{\prime}\right) G\left(k^{\prime}\right) .
$$

In similar way a self-consistent equation for the superconducting order parameter $\varphi$ can be derived under the assumption of Migdal's theorem validity [30]:

$$
\varphi(k)=-\int d k^{\prime} g^{2}\left(k-k^{\prime}\right) D\left(k-k^{\prime}\right) G\left(k^{\prime}\right) G\left(-k^{\prime}\right) \varphi\left(k^{\prime}\right) .
$$

Equations (6) and (7), whose diagrammatic representation is shown in Fig. 3, define a closed set

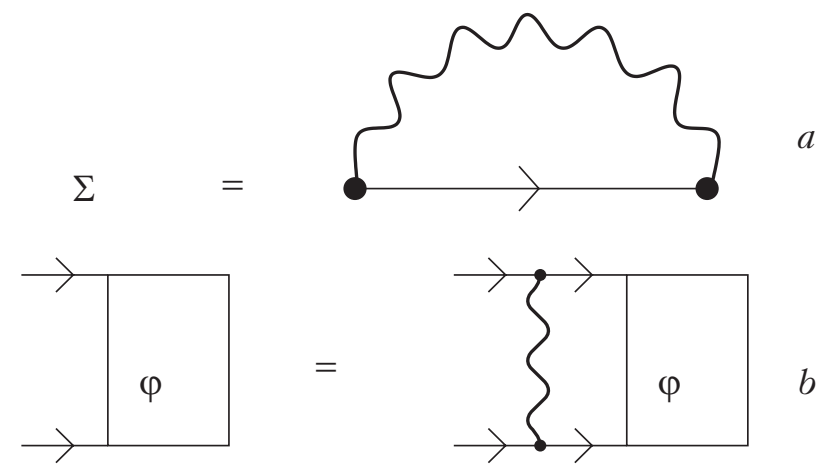

Fig. 3. Self-energy (a) and superconducting pairing (b) of an electron-phonon system in conventional MigdalEliashberg framework. 
of equations provided the phonon spectral function is known. They are thus the basis of the MigdalEliashberg theory of the electron-phonon interaction which was successful to describe many properties of common metal and low-temperature superconductors. For instance the normal state electron-phonon selfenergy at low energy simply reads:

$$
\Sigma=-\lambda \omega
$$

and the coherent part of the Green's function can be written as

$$
G(\mathbf{k}, \omega)=\frac{1}{Z} \frac{1}{\omega-\varepsilon(\mathbf{k}) / Z},
$$

where $Z$ is the electron-phonon renormalization factor $Z=1+\lambda$. Migdal - Eliashberg theory leads thus to an effective renormalization by the factor $1+\lambda$ of many physical quantities of the system, as the Fermi velocity $v_{F}^{*}=v_{F} /(1+\lambda)$, the electron mass $m^{*}=m(1+\lambda)$ or the specific heat $\gamma^{*}=(1+\lambda) 2 \pi^{2} N(0) / 3$ [27].

Similar renormalization arguments permit to understand in a qualitative way the generalization from the BCS equation for $T_{c}$,

$$
T_{c} \propto \omega_{\mathrm{ph}} \exp \left[-\frac{1}{\lambda}\right]
$$

to the McMillan's formula

$$
T_{c} \propto \omega_{\mathrm{ph}} \exp \left[-\frac{1.04(1+\lambda)}{\lambda-\mu^{*}(1+0.62 \lambda)}\right],
$$

valid in strong coupling regime. Taking into account the renormalization of the quasi-particle density of states at the Fermi level $N^{*}(0)=N(0)(1+\lambda)$ as well as of the electron-phonon matrix elements $g^{*}=g /(1+\lambda)$ leads indeed to an effective electron-phonon coupling $\lambda^{*}=\lambda /(1+\lambda)$ which, once plugged in Eq. (10), gives:

$$
T_{c} \propto \omega_{\mathrm{ph}} \exp \left[-\frac{1+\lambda}{\lambda}\right],
$$

which is nothing else that the strong coupling McMillan's formula in the absence of Coulomb repulsion $\mu^{*}$.

It is interesting to note that at the adiabatic ME level all the normal state quantities (renormalized Fermi velocity $v_{F}^{*}$, electronic mass $m^{*}$, specific heat $\gamma^{*}$, quasi-particle spectral weight $Z$, as well as the reduced critical temperature $t_{c}=T_{c} /{ }^{\circ} \mathrm{ph}$, depend on the electron-phonon interaction only through the parameter $\lambda$ which is independent of the atomic mass $M_{\text {at }}$. The isotope coefficient $\alpha_{A}=-d \log A / d \log M_{\mathrm{at}}$ on the normal state properties is thus expected to be zero while $\alpha_{T_{C}}=0.5$.
We would like to make clear once more that all the above results, commonly discussed within the framework of the standard theory of electron-phonon interaction, strongly rely on the adiabatic assumption. When this latter breaks down, a different theory with qualitative different phenomenology is expected.

In order to give just the flavor of the possible deep changes on the physical properties, let us schematize the total electron-phonon vertex function $\Gamma$ in nonadiabatic regime as [31]

$$
\Gamma=g\left[1+\lambda \frac{{ }^{\omega} \mathrm{ph}}{E_{F}}\right],
$$

where the (nonadiabatic) nonzero-order vertex processes were taken into account according with Eq. (5). Equation (13) points out that a sizable renormalization of the electron-phonon vertex function is expected in nonadiabatic systems. The effective phonon mediated electron-electron pairing $\lambda^{*}$ is thus modified in a schematic way in the form $\lambda^{*}=\lambda\left(1+\lambda \omega_{\mathrm{ph}} / E_{F}\right)^{2} /(1+\lambda)$, and the corresponding expression for $T_{c}$ reads [31]:

$$
T_{c} \propto \omega_{\mathrm{ph}} \exp \left[-\frac{1+\lambda}{\lambda\left(1+\lambda \omega_{\mathrm{ph}} / E_{F}\right)^{2}}\right] .
$$

In the extreme nonadiabatic case ${ }^{({ }} \mathrm{ph} / E_{F} \sim 1$ and assuming «normal» values $\lambda=0.4, \omega_{\mathrm{ph}}=700 \mathrm{~K}$, Eq. (14) would thus easily give $T_{c} \simeq 135 \mathrm{~K}$ whereas Eq. (12) would predict $T_{c} \simeq 24 \mathrm{~K}$. We would like to make clear once more that this simple minded generalization should be considered only as indicative since Eq. (5) estimates only the largest magnitude of first order vertex diagrams, not their effective size or sign. In order to determine in a more quantitative way how the onset of nonadiabatic channels on interaction affects the electron-phonon phenomenology, a more detailed analysis is needed.

\subsection{The vertex function beyond Migdal's theorem}

From the above discussion it is clear that a rigorous way of evaluating the nonadiabatic effects is in principle not available since it would imply the full exact solution of the electron-phonon many-body problem. In this situation it is thus of the highest importance to identify physical processes responsible for the nonadiabatic phenomenology and to individuate the more appropriate approaches to deal with them.

Along this view the polaronic picture [32-36] and the nonadiabatic theory of superconductivity [37-39], which we propose for the high- $T_{C}$ compounds, describe two distinct different contexts although they arise from the same electron-phonon interaction [15]. This difference is reflected in the different theoretical tool 
employed in the two cases. A perturbation approach is for instance clearly inadequate to study the polaronic state where ground-state properties are radically different from Fermi liquid ones [36,40]. Nontranslationally invariant states are thus preferably used to account for polaron localization [41,42]. On the other hand a perturbative way appears a natural approach when nonadiabatic effects are expected to modify the Fermi liquid properties in a new nonadiabatic regime without destroying its metallic character [37-39]. In this perspective we believe that a perturbation approach sustained by a diagrammatic representation can provide an useful insight on the relevant physical processes in nonadiabatic systems and a qualitative investigation of their properties. On this basis a quantitative description is therefore beyond the main purposes of our work.

In generalizing the strong coupling theory of Migdal-Eliashberg in nonadiabatic regime, a natural starting point is suggested by Migdal's theorem itself. A controlled perturbative quantity is indeed identified in Eq. (5) by the size of the vertex correction $\lambda \omega_{\mathrm{ph}} / E_{F}$, which can be therefore used as a small expansion parameter. First step of any perturbation analysis of nonadiabatic effects is the explicit evaluation of the first order vertex function $P$, diagrammatically represented in Fig. 4. Its analytical expression reads $[25,26,37]$ :

$$
\begin{aligned}
& P\left(\mathbf{k}, \mathbf{k}^{\prime} ; \omega_{n}, \omega_{m}\right)=-T \sum_{l} \sum_{\mathbf{p}}|g(\mathbf{k}-\mathbf{p})|^{2} D\left(\omega_{n}-\omega_{l}\right) \times \\
& \times G\left(\mathbf{k}^{\prime}+\mathbf{p}-\mathbf{k}, \omega_{m}+\omega_{l}-\omega_{n}\right) G\left(\mathbf{p}, \omega_{l}\right),
\end{aligned}
$$

where $\left(\mathbf{k}, \oplus_{n}\right),\left(\mathbf{k}^{\prime}, \oplus_{m}\right)$ and $\left(\mathbf{p}, \omega_{l}\right)$ are, respectively, the momenta and energies of the incoming, outcoming and internal electrons in Matsubara notations, $G$ and $D$ are the electron and phonon propagators and

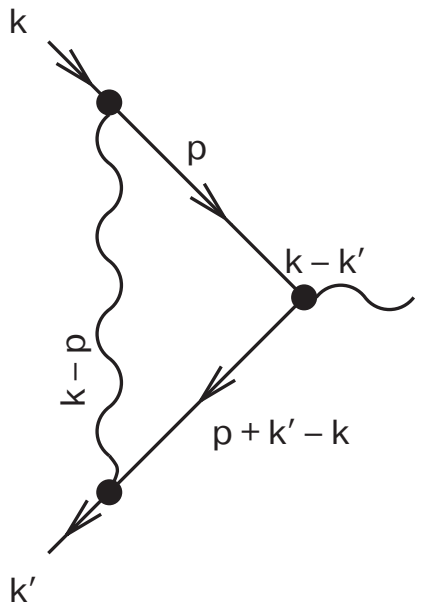

Fig. 4. Feynman's representation of the first order diagram appearing in the nonadiabatic regime. $g(\mathbf{k}, \mathbf{p}) \approx g(\mathbf{k}-\mathbf{p})$ is the electron-phonon matrix element.

The evaluation of the first order vertex diagram beyond Migdal's theorem [Eq. (15)] and its inclusion in systems of electrons scattering with high-energy bosons was previously addressed in the early 80ies by Grabowski and Sham in the context of plasmon based superconductivity [43]. They estimated the vertex function $P$ in the static limit $\lim _{\mathbf{q} \rightarrow 0} \lim _{\omega \rightarrow 0} P(\mathbf{q}, \omega) \quad$ where $\quad \mathbf{q}=\mathbf{k}^{\prime}-\mathbf{k} \quad$ and $\omega=\omega_{m}-\omega_{n}$ are the exchanged momentum and frequency, respectively. In this limit they found that the nonadiabatic electron-phonon interferences described by Eq. (15) disfavor the effective electron-boson attraction, namely $\lim _{\mathbf{q} \rightarrow 0} \lim _{\omega \rightarrow 0} P(\mathbf{q}, \omega)<0$. Similar results are found in later studies based on a local approximation, where the momentum dependence of the vertex function is neglected in favor of averages over the energy variables [44-50]. On this basis the inclusion of vertex diagrams was assumed to be in any case negative with respect to the superconducting pairing. A full momentum dependent analysis shows however that these results are just due to a partial analysis and an effective enhancement of the electron-phonon interaction can actually be induced when the complex momentum structure of the vertex function is properly taken into account [37-39].

In order to evidence this point it is useful to derive an analytical expression of the vertex function which, although approximated, can be used as a guideline to discuss the general momentum-frequency structure. To this aim we consider for the moment the most simplified electron-phonon model containing however all the essential ingredients to the problem. In particular we consider the first order vertex function with bare electron and phonon propagators. Electrons are assumed to interact with a dispersionless Einstein phonon with energy ${ }^{\circ} 0$ through a momentum independent electron-phonon matrix element $g(\mathbf{k}-\mathbf{p})=g$. A half-filled constant density of states will be in addition considered $N(0)=N_{0}$ $[-W \leq \varepsilon \leq W]$ where the half-bandwidth $W$ represents the Fermi energy $W=E_{F}$. Under these assumptions Eq. (15) reads thus [26,37]:

$$
\begin{aligned}
& P\left(\mathbf{k}, \mathbf{k}^{\prime} ; \omega_{n}, \omega_{m}\right)=\frac{2 g^{2}}{\omega_{0}} T \sum_{l} \sum_{\mathbf{p}} \frac{\omega_{0}^{2}}{\omega_{0}^{2}+\left(\omega_{n}-\omega_{l}\right)^{2}} \times \\
& \times \frac{1}{\left[i \omega_{m}+i \omega_{l}-i \omega_{n}-\varepsilon\left(\mathbf{k}^{\prime}+\mathbf{p}-\mathbf{k}\right)\right]\left[i \omega_{l}-\varepsilon(\mathbf{p})\right]} \cdot(16)
\end{aligned}
$$

To obtain an analytical expression the electronic expression can be further linearized around the Fermi level 
$\varepsilon\left(\mathbf{p}+\mathbf{k}^{\prime}-\mathbf{k}\right) \simeq \varepsilon(\mathbf{p})+\mathbf{v}_{p} \cdot\left(\mathbf{k}^{\prime}-\mathbf{k}\right)=\varepsilon(\mathbf{p})+v_{F}|\mathbf{q}| \cos \theta$, where $\theta$ is the angle between $\mathbf{p}$ and $\mathbf{q}=\mathbf{k}^{\prime}-\mathbf{k}$, assuming all the electronic momenta $\mathbf{p}, \mathbf{k}, \mathbf{k}^{\prime}$ to lie on the Fermi surface. The first order vertex diagram results thus to be simply function of the exchanged momentum, $P\left(\mathbf{k}, \mathbf{k}^{\prime} ; \omega_{n}, \omega_{m}\right)=P\left(\mathbf{q} ; \omega_{n}, \omega_{m}\right)$ :

$$
\begin{aligned}
& P\left(\mathbf{q} ; \omega_{n}, \omega_{m}\right)=\frac{2 N(0) g^{2}}{\omega_{0}} T \sum_{l} \frac{\omega_{0}^{2} \omega_{0}^{2}+\left(\omega_{n}-\omega_{l}\right)^{2}}{{ }_{-E_{F}}^{E_{F}} d \varepsilon \times} \\
& \times \int_{-1}^{1} \frac{d \cos \theta}{2\left(i \omega_{m}+i \omega_{l}-i \omega_{n}-\varepsilon-2 E_{F} Q \cos \theta\right)\left(i \omega_{l}-\varepsilon\right)},
\end{aligned}
$$

where $E_{F}=v_{F} k_{F}$ and $Q=|\mathbf{q}| / 2 k_{F}$. It can be shown that the main frequency dependence of the vertex function is only through the exchanged frequency ${ }^{\omega}{ }_{n}-\omega_{m}$. We can therefore set an external frequency to zero ${ }^{\circ}{ }_{n}=0$ so that the exchanged frequency $(1)$ reads just $\omega=\omega_{m}-\omega_{n}=\omega_{m}$. We can now finally derive the following analytic expression for $P$ in the limit of a small $Q$ expansion valid for small exchanged phonon momenta [26,37]:

$$
\begin{aligned}
& P(Q, \omega)=\lambda \frac{\omega_{0}}{2 E_{F} Q} \times \\
& \times\left\{\left[\arctan \left(\frac{\omega}{\omega_{0}}\right)-\arctan \left(\frac{\omega}{\omega_{0}+E_{F}}\right)\right] \arctan \left(\frac{2 E_{F} Q}{\omega}\right)-\right. \\
& \left.-\left[2 E_{F} Q-\omega \arctan \left(\frac{2 E_{F} Q}{\omega}\right)\right] \frac{\left(\omega_{0}+E_{F}\right)\left[\left(\omega_{0}+E_{F}\right)^{2}+2 \omega^{2}\right]}{\left[\left(\omega_{0}+E_{F}\right)^{2}+\omega^{2}\right]^{2}}\right\} .
\end{aligned}
$$

Note that Eq. (18) provides an analytical derivation of Migdal's theorem by evaluating the limit $\lim _{\omega_{0} / E_{F} \rightarrow 0} P$ for generic values of $Q$ and $\omega$. We obtain:

$$
\lim _{\omega_{0} / E_{F} \rightarrow 0} P(Q, \omega)=\lambda \frac{\omega_{0}}{2 E_{F} Q}\left[\frac{\pi}{2} \arctan \left(\frac{|\omega|}{\omega_{0}}\right)-2 Q\right],
$$

which explicitly shows that the first order vertex diagram scales as $\omega_{0} / E_{F}$ in the adiabatic limit.

Equation (18) will be now used as basis to discuss the complex momentum-frequency dependence of the vertex function. In particular we are interested in determining a possible increase or decrease of the effective electron-phonon pairing as due to the onset of nonadiabatic effects. This concept can be quantitatively related to the sign of the vertex correction $P$, where a positive sign $(P>0)$ leads to an enhancement of the effective electron-phonon coupling and a negative sign $(P<0)$ to a reduction [51].

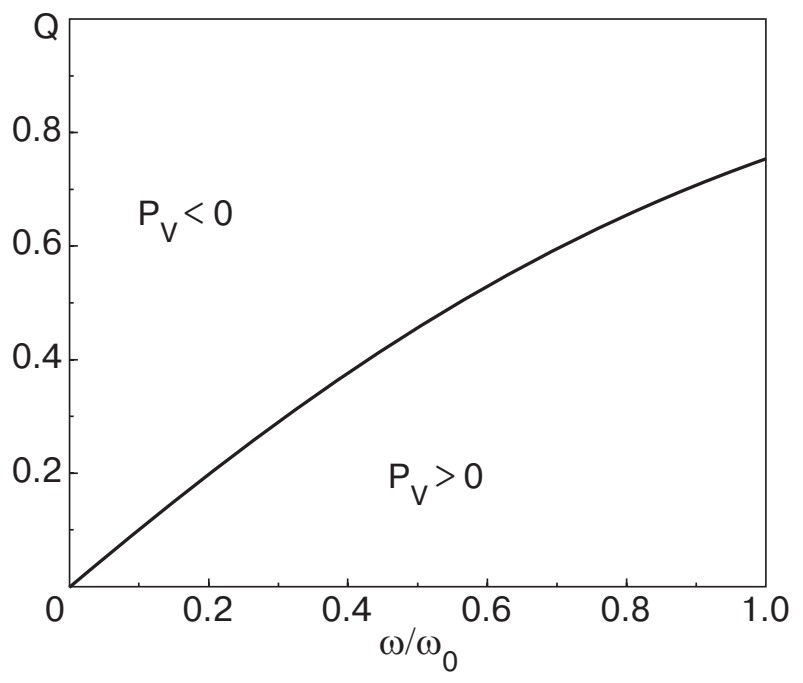

Fig. 5. Sign of the vertex function in the $Q-0$ space for a nonadiabatic system $\left(\omega_{0} / E_{F}=0.5\right)$.

Positive and negative regions of the first order vertex function $P$ are plotted in Fig. 5 in the momentum frequency $Q-\omega$ space for an adiabatic ratio $\oplus_{0} / E_{F}=0.5$. We can see that the total sign of $P$ in not a priori defined but it depends strongly on the specific values of $Q$ and $\omega$. The complex momentum-frequency structure of the vertex function $P$ can be characterized by its static and dynamic limits, respectively, $P^{s}$ and $P^{d}[37,51]$. It can be shown in full generality that:

$$
\begin{aligned}
& P^{s}=\lim _{Q \rightarrow 0} \lim _{\omega \rightarrow 0} P(Q, \omega)<0, \\
& P^{d}=\lim _{\omega \rightarrow 0} \lim _{Q \rightarrow 0} P(Q, \omega)>0,
\end{aligned}
$$

signalizing a nonanalytic point of the vertex function $P$ in $(Q=0, \omega=0)$.

We can now fully understand how the negative sign found in Ref. 43 does not represents the total structure of the vertex diagram but only a limit (static) case. The evaluation of the effects of the nonadiabatic vertex diagrams on the electron-phonon coupling appears thus much more complex and it will depend in general on the specific momentum-frequency region actually probed by the electron-phonon scattering in a particular material.

On one hand, in systems with negligible momentumfrequency dependence of the electron-phonon interaction we can expect for instance that the whole $Q-\infty$ space will be effectively probed. Positive and negative parts of the vertex diagram in this case almost cancel out with a slight dominance of the negative sign. A resulting small reduction of the electron-phonon pairing is thus expected, as confirmed by the analysis in Refs. 44-50. On the other hand materials characte- 
rized by a net predominance of forward ( $\operatorname{small} \mathbf{q}$ ) scattering would mainly probe the small $Q$ positive region of $P$ leading to an effective enhancement of the electron-phonon pairing [37-39].

The crucial role of the electron-phonon momentum structure appears now evident from the above discussion. In this context, approaches as the dynamic meanfield theory (DMFT) or numerical calculations on small clusters are expected to underestimate the importance of the $\mathbf{q}$ dependence and are not suitable for a correct evaluation of the nonadiabatic effects.

A useful tool to study in a controlled way the role of possible $\mathbf{q}(Q)$ momentum selection in the nonadiabatic framework is the introduction of a fictitious cut-off $q_{c}\left(Q_{c}=q_{c} / 2 k_{F}\right)$ which selects small $|\mathbf{q}| \leq q_{c}$ momenta [37-39]. In next sections we shall related on a more compelling ground the cut-off $q_{c}$ to the microscopic properties of the system, e.g., the electronic correlation [52-57]. The electron-phonon matrix elements can be thus modelled as [38]:

$$
|g(\mathbf{q})|^{2}=f\left(q_{c}\right) g^{2} \theta\left(q_{c}-|\mathbf{q}|\right) .
$$

The prefactor $f\left(q_{c}\right)$ can be properly chosen in order to have a total electron-phonon coupling $\lambda$ independent of $q_{c}$, so that the effect of the small momenta selection on the vertex function can be separated from the total phase space reduction which would affect $\lambda$. The prefactor $f\left(q_{c}\right)$ depends thus in principle on the spatial dimension and on peculiar characteristics of the model. For a isotropic system in three dimensions we would have for instance [38]:

$$
|g(Q)|^{2}=\frac{g^{2}}{Q_{c}^{2}} \theta\left(Q_{c}-Q\right),
$$

where we have expressed the exchanged momentum $|\mathbf{q}|$ in the dimensionless variable $Q=|\mathbf{q}| / 2 k_{F}$. Equation (23) ensures that the Fermi surface average of the phonon mediated electron-electron interaction is constant $\left\langle|g(Q)|^{2}\right\rangle_{F S}=g^{2}$. In this way the electronphonon coupling constant $\lambda$ and the cut-off parameter $Q_{c}$ can be considered as two independent free variables.

On the basis provided by Fig. 5 and by Eq. (18) we can now qualitatively investigate the role of a small momenta selection in the context of a nonadiabatic electron-phonon interaction. This issue will be parametrized as function of the cut-off $Q_{c}$, where $Q_{c} \simeq 1$ represents a shapeless electron-phonon interaction in the momentum space and $Q_{c}<<1$ a marked forward scattering predominance which could be representative of strongly correlated systems [52-57]. In order to evaluate the effect of the inclusion of the first order vertex function $P$ in a generic phonon mediated property (electronic self-energy, superconducting pairing) we consider a weighted average of $P(Q, \omega)$ in the whole momentum-frequency space, $P^{\text {av }}\left(Q_{c}\right)$ [37]. Weighting factors will be the momentum dependence electron-phonon matrix elements as given by Eq. (23), which would take into account a possible small momentum selection, and a simple Lorentzian in frequency $\omega_{0}^{2} /\left(\omega_{0}^{2}+\omega^{2}\right)$ to simulate the presence of a phonon propagator with characteristic energy $\omega_{0}$ which would be always connected to the electron-phonon vertex function.

The dependence of the weighted average vertex function on the adiabatic ratio $\omega_{0} / E_{F}$ is shown in Fig. 6 for different values of the cut-off $Q_{c}$. Note the strong enhancement for small values of $Q_{c}$ and the fact that a significant magnitude of $P^{\mathrm{av}}\left(Q_{c}\right)$ is already appreciable for relatively small values of $\omega_{0} / E_{F}$.

According to this preliminary qualitative analysis we can then identify a physical regime characterized by a finite adiabatic ratio $\omega_{0} / E_{F} \neq 0$ and small momentum phonon scattering $\left(Q_{c}<1\right)$ where the nonadiabatic effects induced by the breakdown of Migdal's theorem could lead to a remarkable enhancement of the electron-phonon pairing. Guided by these results a rigorous generalization of the theory of superconductivity in nonadiabatic regime $[38,39]$ will be presented in the next section. We are going to see that the enhancement of the superconducting pairing suggested by the above discussion is actually confirmed by a numerical accurate analysis. The normal and superconducting state phenomenology of the nonadiabatic systems will be also investigated.

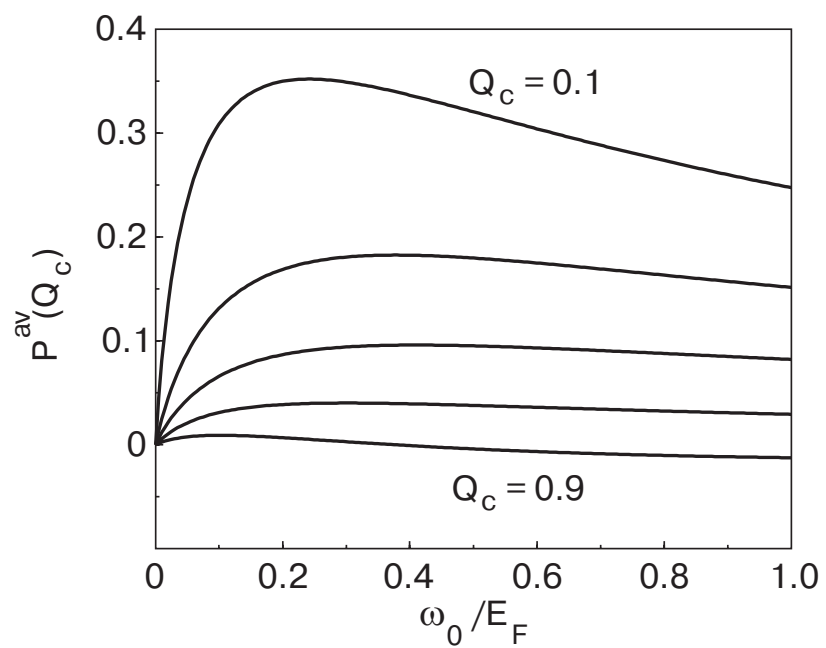

Fig. 6. Momentum-frequency average of the vertex diagram $P^{\mathrm{av}}\left(Q_{C}\right)$ as function of the adiabatic parameter $\omega_{0} / E_{F}$ for different values of the momentum cut-off $Q_{c}$ (from the top to the bottom): $Q_{c}=0.1,0.3,0.5,0.7,0.9$, and for $\lambda=1$. 


\section{Nonadiabatic theory of superconductivity and normal state}

In Sec. 2 we have briefly mentioned the possibility to build a perturbative theory in regime of strong coupling $\lambda \sim 1$ on the basis of a small $\omega_{0} / E_{F}$ expansion. The most straightforward way to achieve this aim is the use of the functional formalism based on the Baym-Kadanoff technique [58,59] to derive a conserving scheme which links the superconducting and normal state properties to a self-consistent evaluation of the normal state self-energy in nonadiabatic regime. In terms of Feynman's diagrams a convenient starting point is the skeleton formalism where each graphical element represents fully renormalized quantities $[28,29]$.

\subsection{One-particle self-energy}

In this framework the diagrammatic generalization of the electron-phonon self-energy at the first order in a $\omega_{0} / E_{F}$ expansion is depicted in Fig. 7. Retaining only the first term on the right side is equivalent to the so called noncrossing approximation (NCA) where nonadiabaticity would be taken into account only through finite bandwidth effects. More importance is attached to the second term on the right side which explicitly contains the first order vertex diagram arising from the breakdown of Migdal's theorem. Its analytic expression can be written in the compact form as $[11,38,39]$ :

$$
\begin{aligned}
& \Sigma\left(\mathbf{k}, \omega_{n}\right)=T \sum_{\mathbf{k}^{\prime}, m} D\left(\omega_{n}-\omega_{m}\right)\left|g\left(\mathbf{k}-\mathbf{k}^{\prime}\right)\right|^{2} \times \\
& \times\left[1+P\left(\mathbf{k}, \mathbf{k}^{\prime} ; W_{n}, W_{m}\right)\right] G\left(\mathbf{k}^{\prime}, W_{m}\right),
\end{aligned}
$$

where we have introduced the renormalized Matsubara frequencies $i W_{n}=i \omega_{n}-\Sigma\left(\omega_{n}\right), D$ is the generic phonon propagator coupled to the electrons through the electron-phonon Eliashberg function $\alpha^{2} F(\omega)$ [27,60,61]:

$$
D\left(\omega_{n}-\omega_{m}\right)=\frac{2}{\lambda} \int \frac{\omega \alpha^{2} F(\omega)}{\omega^{2}+\left(\omega_{n}-\omega_{m}\right)^{2}} d \omega
$$

and $P$ is the first order vertex function:

$$
\begin{aligned}
& P\left(\mathbf{k}, \mathbf{k}^{\prime} ; W_{n}, W_{m}\right)=T \sum_{\mathbf{p}, l}|g(\mathbf{k}-\mathbf{p})|^{2} D\left(\omega_{n}-\omega_{l}\right) \times \\
& \times G\left(\mathbf{p}+\mathbf{k}^{\prime}-\mathbf{k}, W_{l-n+m}\right) G\left(\mathbf{p}, W_{l}\right) .
\end{aligned}
$$

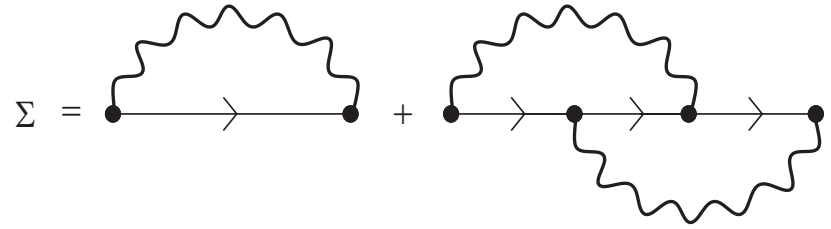

Fig. 7. Nonadiabatic electron-phonon self-energy including the first order vertex diagram arising from the breakdown of Migdal's theorem.

In an isotropic system the angular dependence of observable quantities is negligible and it can be dropped. We can therefore replace the self-energy in Eq. (24) and the whole electron-phonon matrix elements in Eq. (26), including the vertex corrections, with their averages over the Fermi surface $[11,38,39]$ :

$$
\begin{aligned}
& \Sigma\left(\mathbf{k}, \omega_{n}\right) \rightarrow\left\langle\Sigma\left(\mathbf{k}, \omega_{n}\right)\right\rangle_{F S}=\Sigma\left(\omega_{n}\right), \\
\left|g\left(\mathbf{k}-\mathbf{k}^{\prime}\right)\right|^{2}\left[1+P\left(\mathbf{k}, \mathbf{k}^{\prime} ; W_{n}, W_{m}\right)\right] \rightarrow & \rightarrow\left\langle\left[1+P\left(\mathbf{k}, \mathbf{k}^{\prime} ; W_{n}, W_{m}\right)\right]\right\rangle_{F S}= \\
= & g^{2}\left[1+P\left(Q_{c} ; W_{n}, W_{m}\right)\right] .
\end{aligned}
$$

Equations (24), (27) and (28) define a self-consistent expression for the self-energy which can be written in the compact form:

$$
\begin{aligned}
& W_{n}=\omega_{n}-2 \lambda T \sum_{m}\left[1+P\left(Q_{c} ; W_{n}, W_{m}\right)\right] \times \\
& \times D\left(\omega_{n}-\omega_{m}\right) \arctan \left(\frac{E_{F}}{W_{M}}\right),
\end{aligned}
$$

and which can be numerically solved once the momentum averaged vertex function $P\left(Q_{c} ; W_{n}, W_{m}\right)$ is known. An explicit expression of $P\left(Q_{c} ; W_{n}, W_{m}\right)$ can be derived within the same approximations employed in Sec. 2. However, because of the self-consistent renormalization of the internal Green's functions in Eq. (26), we cannot obtain now an analytical form for $P\left(Q_{c} ; W_{n}, W_{m}\right)$ since it would involve a sum over the Matsubara frequencies. A longsome derivation of $P\left(Q_{c} ; W_{n}, W_{m}\right)$, which is essentially based on a small $Q_{c}$ expansion, can be found in Refs. 62, 63 where we refer for more details. We report here therefore only the final result: 


$$
\begin{aligned}
P\left(Q_{c} ; W_{n}, W_{m}\right) & =\lambda T \sum_{l} D\left(\omega_{n}-\omega_{l}\right)\left(B(n, m, l)+\frac{A(n, m, l)-B(n, m, l)\left[W_{l}-W_{l-n+m}\right]^{2}}{\left(2 E_{F} Q_{c}^{2}\right)^{2}} \times\right. \\
& \left.\times\left\{\sqrt{1+\left(\frac{4 E_{F} Q_{c}^{2}}{W_{l}-W_{l-n+m}}\right)^{2}}-1-\ln \left[\frac{1}{2} \sqrt{1+\left(\frac{4 E_{F} Q_{c}^{2}}{W_{l}-W_{l-n+m}}\right)^{2}}+\frac{1}{2}\right]\right\}\right),
\end{aligned}
$$

where

$$
\begin{aligned}
& A(n, m, l)=\left(W_{l}-W_{l-n+m}\right)\left[\arctan \left(\frac{E_{F}}{W_{l}}\right)-\arctan \left(\frac{E_{F}}{W_{l-n+m}}\right)\right], \\
& B(n, m, l)=\left(W_{l}-W_{l-n+m}\right) \frac{E_{F} W_{l-n+m}}{\left[E_{F}^{2}+W_{l-n+m}^{2}\right]^{2}}-\frac{E_{F}}{E_{F}^{2}+W_{l-n+m}^{2}} .
\end{aligned}
$$

Equations (29)-(32) define now a closed set of equations which determine in an unambiguous way all the one-particle properties of a nonadiabatic system. The nonadiabatic equations are valid for any generic electron-phonon spectrum described by the Eliashberg function $\alpha^{2} F(\omega)$. They will be thus the basis for investigating the characteristic features in the normal state of nonadiabatic systems. For practical purposes we shall consider in the following, unless better specified, a single Einstein phonon spectrum, which reproduces however all the relevant nonadiabatic characteristics. As we are going to see, interesting new physics is induced by the onset of new channels of interaction in the nonadiabatic regime.

A natural quantity to be investigated in this framework is the effective electronic mass $m^{*}$ which is renormalized by the electron-phonon interaction. Hallmark result of the conventional Migdal-Eliashberg theory is the simple relation between the effective mass $m^{*}$ and the unrenormalized one $m$ : $m^{*}=m(1+\lambda)$ [27]. Straightforward consequence of this relation is the absence of any isotope effect on $m^{*}$, since the electron-phonon coupling constant $\lambda$ can be shown not to depend on the atomic masses. This prediction is indeed verified in all common metals.

The effective mass $m^{*}$ in our context can be easily obtained from Eqs. (29)-(32) as a simple limit of the renormalization function $Z\left(i \omega_{n}\right)=1-\Sigma\left(i \omega_{n}\right) / i \omega_{n}$ [11]:

$$
\frac{m^{*}}{m}=\lim _{i \omega_{n} \rightarrow 0} Z\left(i \omega_{n}\right)=\lim _{i \omega_{n} \rightarrow 0}\left[1-\frac{\sum\left(i \omega_{n}\right)}{i \omega_{n}}\right] .
$$

In Fig. 8 we plot the quantity $Z\left(i \omega_{n}\right)$, calculated in nonadiabatic regime $\left(\omega_{0} / E_{F}=0.2\right)$ by including the first order vertex diagram as in Fig. 7 , as function of $\omega_{n}$ for $\lambda=1.0$ [11]. A marked dip around $\omega_{n}=0$ is observed in the nonadiabatic theory (solid lines) which is absent when the vertex diagram is omitted (dashed line, noncrossing approximation). This feature can be understood by considering that at $\omega_{n}=0$ the electron mass renormalization factor $Z$ is mostly modified by the static limit of the vertex function, $\lim _{W_{m} \rightarrow W_{n}} P\left(Q_{c} ; W_{n}, W_{m}\right)$, which was previously shown to be negative.

Another interesting feature appearing in the nonadiabatic regime is an effective dependence of the electronic mass $m^{*}$ on the adiabatic ratio and consequently on the atomic mass $M_{\text {at }}$. This concept leads thus to a generalization of the standard Migdal-Eliashberg expression for $m^{*}$ in nonadiabatic systems:

$$
m^{*}=m f_{m *}\left(\lambda, \frac{\omega_{0}}{E_{F}}\right)
$$

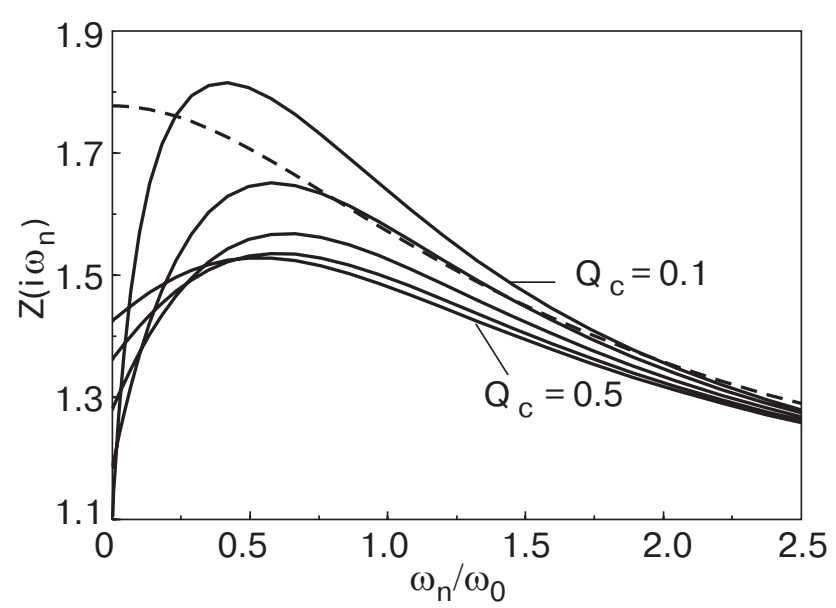

Fig. 8. Renormalization function $Z\left(i \omega_{n}\right)$ for $\omega_{0} / E_{F}=0.2$ and $\lambda=1.0$. Solid lines: nonadiabatic theory with $Q_{c}=0.1,0.2, \ldots, 0.5$. Dashed line: noncrossing approximation with no vertex diagram. 


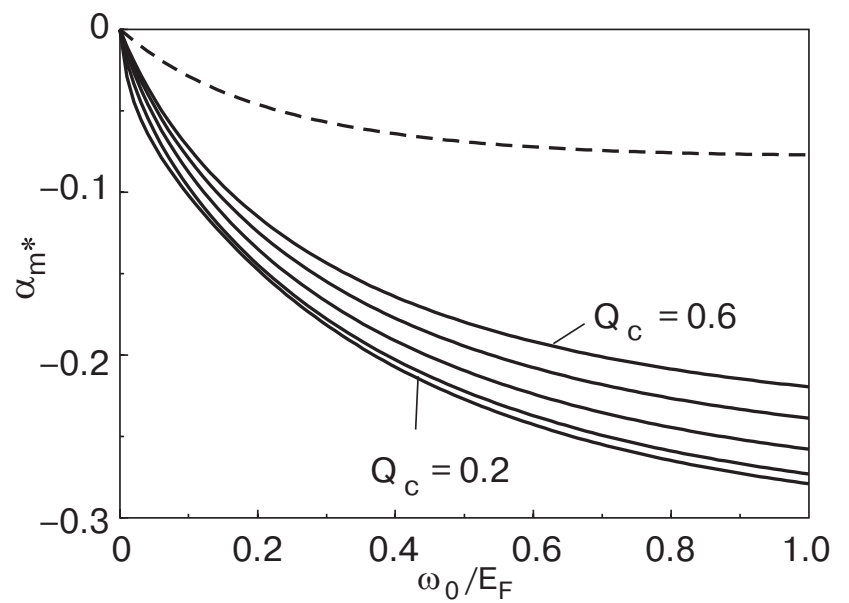

Fig. 9. Isotope coefficient $\alpha_{m} *$ on the effective electronic mass $m^{*}$ calculated for $\lambda=1$. Solid lines: nonadiabatic theory for $Q_{c}=0.2,0.3, \ldots, 0.6$. Dashed line: noncrossing approximation with no vertex diagrams.

where $\lim _{\omega_{0} / E_{F} \rightarrow 0} f_{m^{*}}\left(\lambda, \omega_{0} / E_{F}\right)=1+\lambda$. The explicit dependence of the effective electronic mass on the adiabatic ratio is reflected in a finite isotope effect on $m^{*}$. In Fig. 9 we report the isotope coefficient $\alpha_{m^{*}}$ as function of the adiabatic parameter ${ }^{\oplus} 0 / E_{F}$ [11]. Solid lines correspond to the nonadiabatic theory for different values of $Q_{c}$, dashed line to the noncrossing approximation where nonadiabaticity was retained only through finite bandwidth effects. A finite negative isotope effect is recovered in the nonadiabatic regime, mainly due to the onset of nonadiabatic channels of interaction (compared with the dashed line). These results were later confirmed by DMFT calculations [15]. Note moreover that $\alpha_{m^{*}}$ does not show in the whole region of $\omega_{0} / E_{F}$ a crucial dependence on $Q_{c}$. This is again due to the particular limit of the vertex function probed by the electronic mass $m^{*}$ which is not significantly affected by the small $|\mathbf{q}|$ selection. The prediction of a finite and negative isotope effect on $m^{*}$ is of the highest importance with respect to the experimental observation of an isotope effect on the penetration depth $\lambda_{L}(0)$ in cuprates which is indeed thought to stem from a corresponding isotope effect on the electronic mass $m^{*}$.

\subsection{Superconducting instability}

The study of the nonadiabatic effects on the superconducting instability and on the resulting critical temperature $T_{c}$ appears of fundamental importance in the light of the specific properties of the high- $T_{c}$ materials, that we have seen to present small Fermi energies, and of the previous qualitative discussion in Sec. 2 which suggested that an enhancement of the superconducting pairing is achievable in the nonadiabatic regime under favorable conditions like the predominance of forward scattering [37].

The formal derivation of the nonadiabatic theory of superconductivity follows essentially the same procedure developed for the normal state. The explicit equations can be determined by the requirement of a conserving theory consistent with the self-energy $\Sigma$ depicted in Fig. 7. Technical steps are the introduction of an external field $h^{\varphi}$ coupled with the superconducting condensate $\varphi \sim\left\langle c^{\dagger} c^{\dagger}\right\rangle$ and the functional derivative of $\Sigma$ with respect to $h^{\varphi}$ in the spirit of the Baym - Kadanoff formalism.

The practical derivation is accomplished in the clearest way in terms of the diagrammatic representation. The graphical expression of the superconducting instability equation is thus obtained by replacing in all the possible combinations one of the electronic lines of Fig. 7 with an anomalous propagator which can be in its turn expressed as function of the superconducting order parameter $\varphi$ and of two normal state propagators with $\left(\mathbf{k}, \oplus_{n}, \rightarrow\right)$ and $\left(-\mathbf{k},-\oplus_{n}, \downarrow\right)$ quantum numbers $[38,39]$, respectively. We obtain then a self-consistent equation for the superconducting order parameter $\varphi$ as diagrammatically shown in Fig. 10.

We can see that the failure of Migdal's theorem gives rise to two vertex diagrams, which have been already widely discussed, and to one so-called «cross» term $[38,39]$. In similar way with the normal state self-energy, we can write down the explicit self-consistent equation for $\varphi$ :

$$
\begin{aligned}
& \varphi\left(\mathbf{k}, \omega_{n}\right)=T \sum_{\mathbf{k}^{\prime}, m}\left\{D\left(\omega_{n}-\omega_{m}\right)\left|g\left(\mathbf{k}-\mathbf{k}^{\prime}\right)\right|^{2} \times\right. \\
& \left.\times\left[1+2 P\left(\mathbf{k}, \mathbf{k}^{\prime} ; W_{n}, W_{m}\right)\right]-C\left(\mathbf{k}, \mathbf{k}^{\prime} ; W_{n}, W_{m}\right)\right\} \times \\
& \times G\left(\mathbf{k}^{\prime}, W_{m}\right) G\left(-\mathbf{k}^{\prime},-W_{m}\right) \varphi\left(\mathbf{k}^{\prime}, \omega_{m}\right),
\end{aligned}
$$

where the cross function $C$ is defined as:

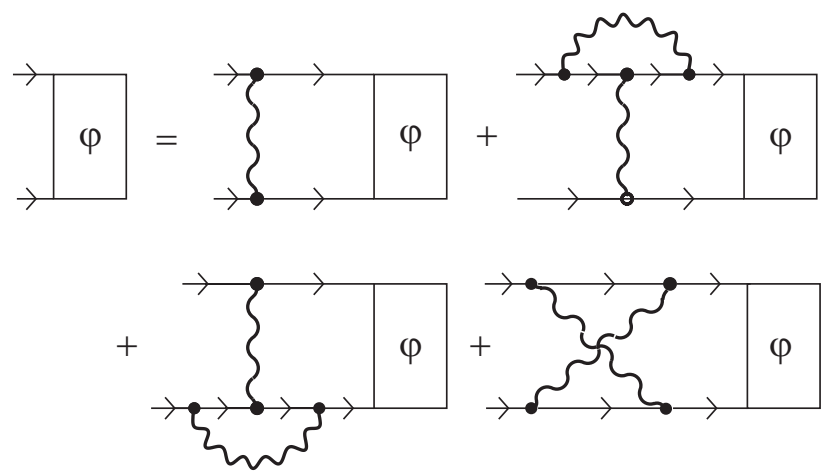

Fig. 10. Self-consistent equation for the superconducting order parameter $\varphi$ in the nonadiabatic theory. 


$$
\begin{aligned}
& C\left(\mathbf{k}, \mathbf{k}^{\prime} ; W_{n}, W_{m}\right)=T \sum_{\mathbf{p}, l}|g(\mathbf{k}-\mathbf{p})|^{2}\left|g\left(\mathbf{p}-\mathbf{k}^{\prime}\right)\right|^{2} \times \\
& \times D\left(\omega_{n}-\omega_{l}\right) D\left(\omega_{l}-\omega_{m}\right) \times \\
& \times G\left(\mathbf{p}-\mathbf{k}-\mathbf{k}^{\prime}, W_{l-n-m}\right) G\left(\mathbf{p}, W_{l}\right) .
\end{aligned}
$$

For an isotropic superconductivity (we shall discuss later $d$-wave symmetry), we can also average the superconducting order parameter $\varphi$ on the Fermi surface. We end up with the resulting equation for the superconducting instability in Matsubara frequencies:

$\varphi_{n}=2 \lambda T_{c} \sum_{m}\left\{\left[1+2 P\left(Q_{c} ; W_{n}, W_{m}\right)\right] \times\right.$

$\left.\times D\left(\omega_{n}-\omega_{m}\right)+C\left(Q_{c} ; W_{n}, W_{m}\right)\right\} \frac{\varphi_{m}}{W_{m}} \arctan \left(\frac{E_{F}}{W_{m}}\right)$.

The explicit derivation of the cross function can be carried on in similar way with the vertex $P$ to obtain [62]:

$$
\begin{aligned}
& C\left(Q_{c} ; W_{n}, W_{m}\right)=\lambda^{2} T_{c} \sum_{l} D\left(\omega_{n}-\omega_{l}\right) D\left(\omega_{l}-\omega_{m}\right) \times \\
& \times\left\{2 B(n,-m, l)+\arctan \left(\frac{4 E_{F} Q_{c}^{2}}{W_{l}-W_{l-n-m}}\right) \times\right. \\
& \left.\times \frac{A(n,-m, l)-B(n,-m, l)\left[W_{l}-W_{l-n-m}\right]^{2}}{2 E_{F} Q_{c}^{2}\left[W_{l}-W_{l-n-m}\right]^{2}}\right\}
\end{aligned}
$$

where the functions $A(n, m, l)$ and $B(n, m, l)$ are the same ones defined in Eqs. (31), (32).

Equation (37), together with Eq. (29), evaluated at $T=T_{c}$, defines the nonadiabatic theory of superconductivity which can be employed for numerical calculations. We are now in the position to investigate with a full numerical solution the role of the opening of nonadiabatic pairing channels on the superconducting critical temperature.

In Fig. 11 we show the critical temperature $T_{c}$ as function of the adiabatic parameter $\omega_{0} / E_{F}$ for different values of the dimensionless momentum cut-off $Q_{c}$ and for $\lambda=0.7$. The case $\omega_{0} / E_{F}=0$ corresponds to the Migdal's limit. Interestingly, nonadiabatic effects can affect in different ways the superconducting pairing, resulting in an increase or reduction of $T_{c}$ depending on the microscopic details of the system (the ratio $\omega_{0} / E_{F}=0$, the degree of the electronic correlation $\left.Q_{c}, \ldots\right)$. We can note however that the intuitive idea that strong electronic correlations (small $Q_{c}$ 's) can favor superconductivity by selecting positive regions of the vertex function is sustained by the numerical calculations [38,39]. In Fig. 11 a marked enhancement of $T_{c}$ with respect to the conventional

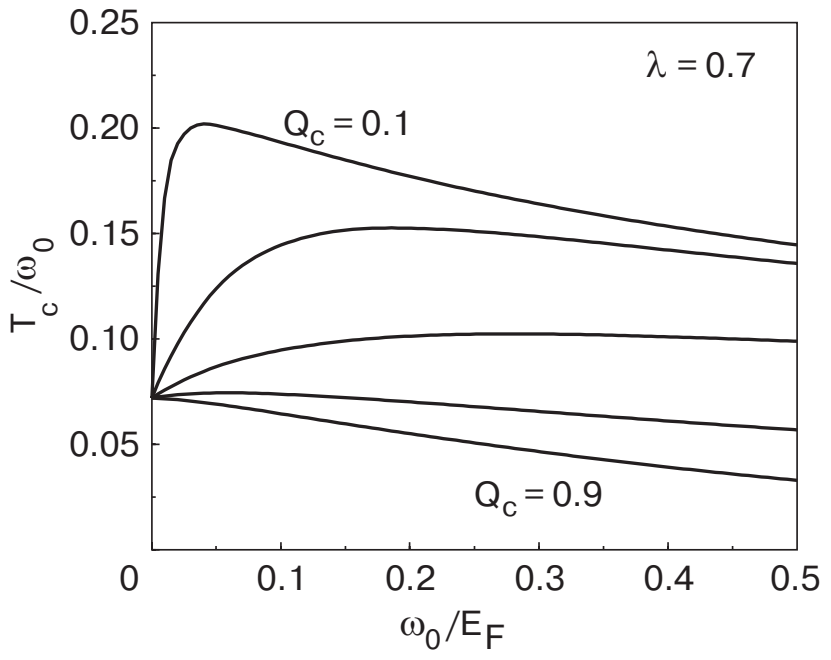

Fig. 11. Superconducting critical temperature $T_{C}$ in the nonadiabatic theory as function of the ratio $\omega_{0} / E_{F}$ for $\lambda=0.7$ and different values of $Q_{c}$ (from the top to the bottom line): $Q_{c}=0.1,0.3,0.5,0.7,0.9$.

Migdal-Eliashberg theory is shown for relatively small values of $Q_{c}$.

We can also easily compute from Eqs. (29), (37) the isotope coefficient on $T_{c}$. The results are shown in Fig. 12. We can note some interesting features. First of all a reduced isotope effect $\alpha_{T_{c}}<0.5$ can be found as result of the nonadiabatic pairing (note that in the present analysis no Coulomb repulsion was taken into account so that should be strictly equal to 0.5 in the Migdal-Eliashberg framework). At the same time an isotope coefficient $\alpha_{T_{c}}>0.5$ can be also observed, which is a quite unconventional feature. It is interest-

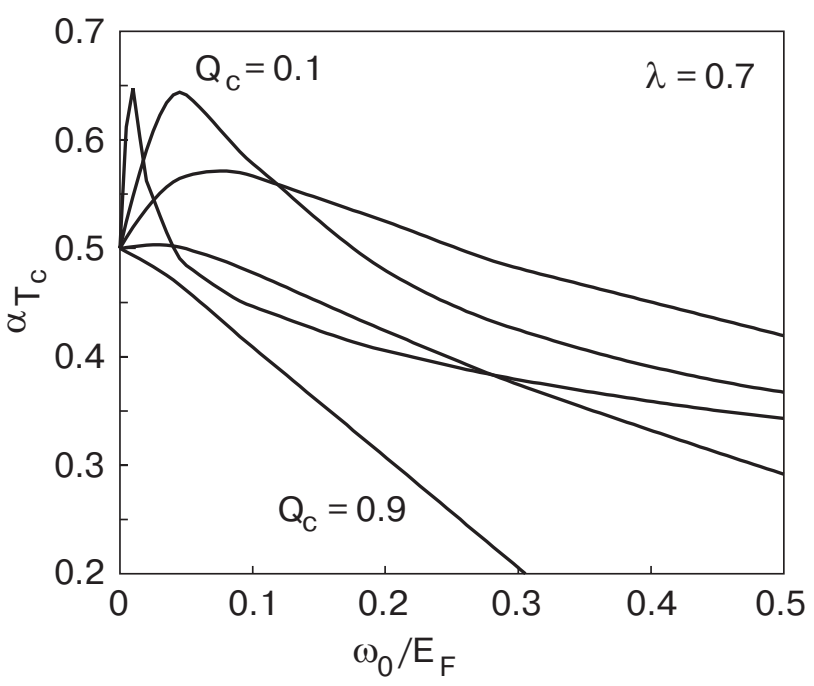

Fig. 12. Isotope coefficient $\alpha_{T_{C}}$ on $T_{C}$ in the nonadiabatic theory as function of the adiabatic ratio. Same values of $\lambda$ and $Q_{c}$ as in previous figure. Smaller values of $Q_{c}$ correspond to lines with steeper initial slope. 
ing to note also that the stronger variations of $\alpha_{T_{C}}$ are predicted for small $Q_{c}$ 's, i.e., when higher $T_{c}$ 's are recovered.

The Nambu formalism permits to generalize the nonadiabatic theory of superconductivity also below the critical temperature $T_{c}$ to evaluate zero temperature quantities as for instance the superconducting gap $\Delta$. The formal derivation is not difficult but quite tedious and we refer to Ref. 64 for technical details. An important point is that the vertex and cross functions, as well the corresponding nondiagonal quantities in the Nambu space, need to be evaluated in the presence of the superconducting gap which partially reduces the nonanaliticity of these function at the $(\mathbf{q}=0, \omega=0)$ point. The moment-frequency structure of the vertex function in the superconducting state is report in Fig. 13 which shows that the net result of the opening of the superconducting gap is to reduce the positive region of the vertex function and to increase the negative one. A direct consequence of this effect is that the effective superconducting pairing in the superconducting state at $T=0$ is smaller than the one at $T=T_{c}$, so that the ratio $2 \Delta / T_{c}$, for a given $\lambda$, is smaller in the nonadiabatic framework than in ME theory [64].

The scenario here outlined shows the breakdown of the conventional picture of the superconducting and normal state properties in nonadiabatic systems. In particular it is clear that the inclusion of higher order vertex terms arising from the violation of Migdal's

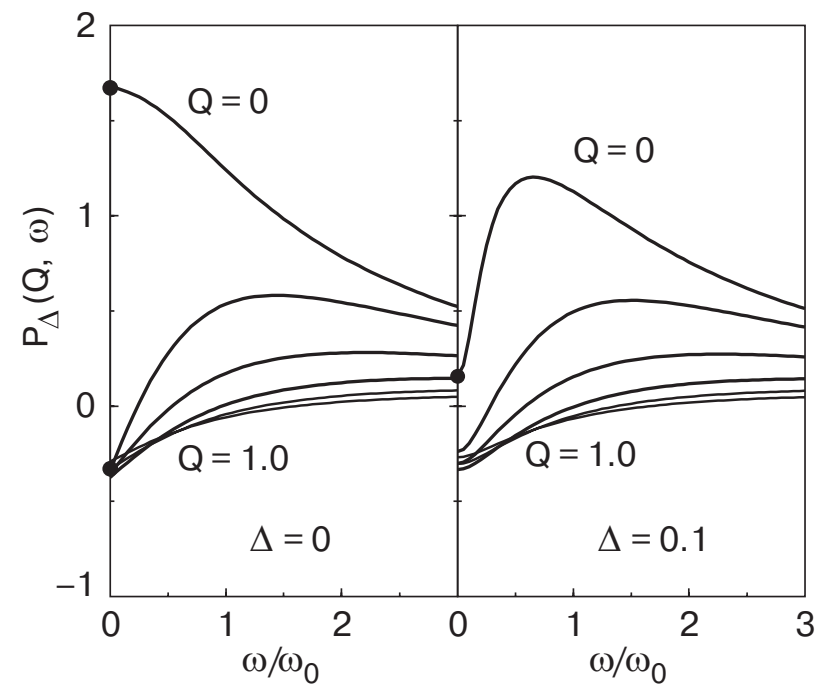

Fig. 13. Frequency structure of the vertex function for different values of the exchanged momentum: (from top line to the bottom) $Q=0,0.2,0.4, \ldots, 1.0$. The adiabatic parameter is here set $\omega_{0} / E_{F}=0.2$. Left panel refers to the normal state $(\Delta=0)$, right panel to the superconducting state $\left(\Delta=0.1 \omega_{0}\right)$. Filled circles mark the static and dynamic limits in the normal and superconducting state. theorem does not lead to an effective renormalization of the electron-phonon coupling $\lambda$ but defines a new physical regime where conventional microscopic parameters $\left(\lambda, \omega_{0}, \ldots\right)$ can give rise to unconventional features [65]. This is evident for instance by looking at the isotope effects on $T_{c}$ and on $m^{*}$ which would be $\alpha_{T_{c}}=0.5$ and $\alpha_{m^{*}}=0$, respectively, if just a renormalization of $\lambda$ would be involved. This is clearly shown also by the comparison between Figs. 9 and 12 where it is clear that the intrinsic dependence on the microscopic parameters $\left(\lambda, \omega_{0}, Q_{c}, \ldots\right)$ can be very different according which physical quantity is considered.

Additional evidence of the breakdown of the conventional phenomenology in nonadiabatic superconductors is provided by the anomalous dependence of the critical temperature on nonmagnetic impurities [62]. A well-known theorem in the conventional theory of superconductivity states indeed that scattering from disorder or nonmagnetic impurities should have a strictly zero effect on $T_{c}$ in isotropic $s$-wave superconductors in adiabatic regime [66]. A violation of this theorem however has been experimentally observed in fullerides and in electron-doped $s$-wave cuprates. The nonadiabatic theory of superconductivity provides a natural framework to explain this anomalous behavior as shown in Fig. 14 where we show the strong reduction of the critical temperature $T_{c}$ as function of the impurity scattering rate $\Gamma[62]$. In agreement with the previous data on $T_{c}, m^{*}, \alpha_{T_{c}}, \alpha_{m^{*}}$, this unconventional reduction is more marked for small $Q_{c}$ 's where nonadiabatic effects are shown to be stronger.

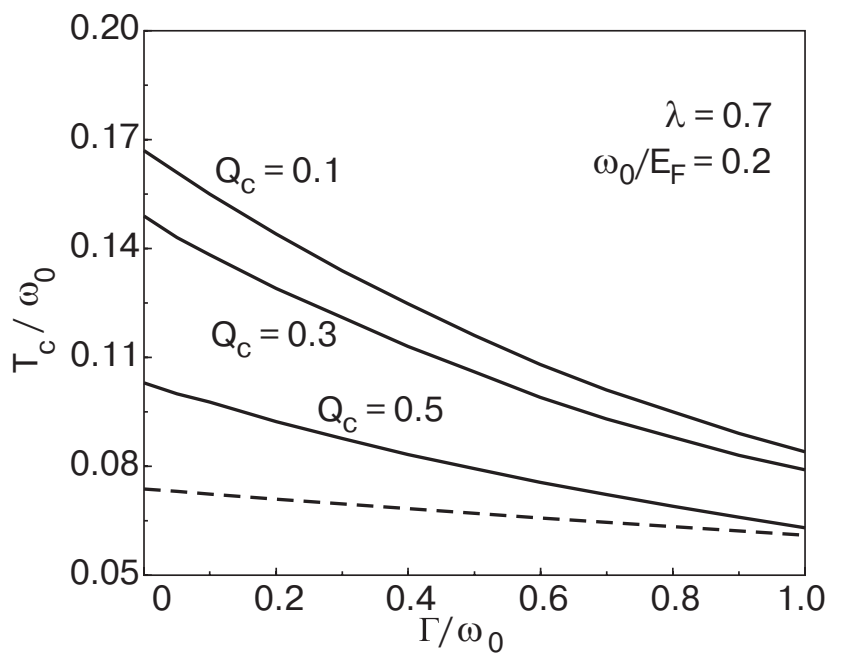

Fig. 14. Critical temperature as a function of the impurity scattering rate $\Gamma$ for different values of $Q_{c}$ in the nonadiabatic theory. The dashed line corresponds to the noncrossing approximation with no vertex contribution. 


\subsection{Phenomenology in normal state: the Pauli spin susceptibility}

In the previous section we have stressed the importance to predict electron-phonon anomalous effects in the nonadiabatic scenario which are not expected within the ME theory and which can be employed as direct experimental tests. One of them was the the effective electron mass $m^{*}$ which at the ME level depends only on $\lambda$ but not on the phonon frequencies so that no isotope effect should appear. Along this line the Pauli susceptibility $\chi_{P}$ is another promising quantity to evidence nonadiabatic electron-phonon effects since it is expected to be completely unaffected by the electron-phonon coupling within the conventional Migdal-Eliashberg theory [27]. Following the Baym - Kadanoff formalism $[58,59]$ one can write a general expression for $\chi_{P}$ :

$$
\chi_{P}=-2 \mu_{B}^{2} T \sum_{\mathbf{k}, n} G^{2}\left(\mathbf{k}, W_{n}\right) \Gamma_{\chi}\left(\mathbf{k}, W_{n}\right),
$$

where $\mu_{B}$ is the Bohr magneton and $\Gamma_{\chi}$ is the total spin vertex function. It can be shown that the electron-phonon interaction in the Green's functions $G$ and in the spin vertex $\Gamma_{\chi}$ cancels out in the adiabatic limit since the electron-phonon self-energy $\Sigma^{e p}$ in the presence of a magnetic field $H$ has nonzero contributions only at a nonadiabatic level [67-69]:

$$
\lim _{H \rightarrow 0} \Sigma^{e p}=H O\left(\omega_{0} / E_{F}\right) .
$$

Hence, any evidence of electron-phonon effects on the Pauli spin susceptibility would be a direct proof of a nonadiabatic electron-phonon coupling $[63,69]$.

The proper inclusion of the electron-phonon interaction in Eq. (39) requires the evaluation of the spin vertex function $\Gamma_{\gamma}$ in nonadiabatic regime [63]. This can be done following the diagrammatic scheme previously discussed for the two particle superconducting response. The pictorial expression of the spin vertex $\Gamma_{\gamma}$ including electron-electron exchange interaction and the nonadiabatic electron-phonon coupling is shown in Fig. 15. Explicit expressions for the vertex and the cross diagrams appearing in Fig. 15 can be derived in similar way as in the Cooper pairing case, by using the vertex and cross functions defined in Eqs. (30), (38). Numerical calculations can be therefore performed by the self-consistent solution of the Fig. 15 and of the frequency renormalization equation.

In Fig. 16 we plot the total spin susceptibility (electron-electron + electron-phonon scattering) as function of the electron-phonon coupling and of the adiabatic parameter $\omega_{0} / E_{F}$ for zero temperature [63]. Dashed lines are the results obtained within the noncrossing approximation without electron-phonon ver-

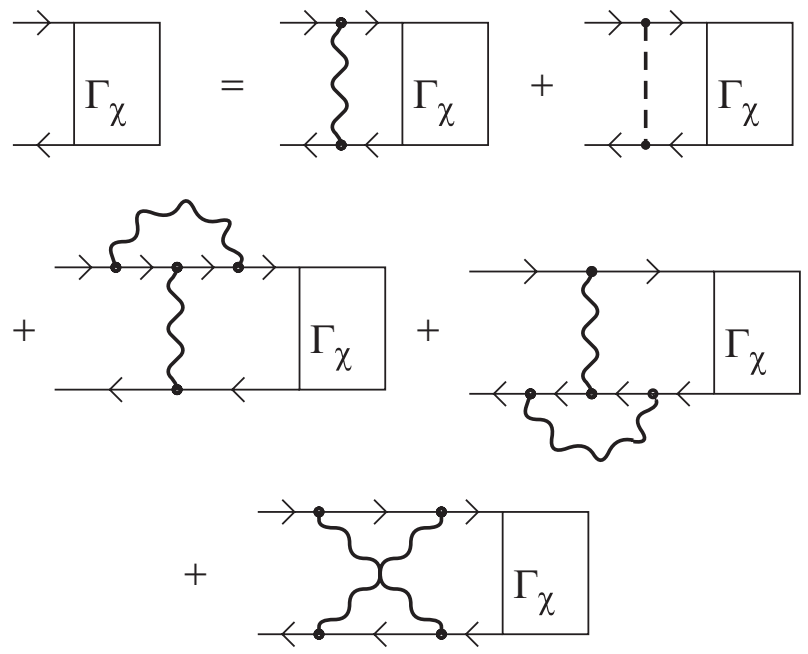

Fig. 15. Diagrammatic representation of the spin vertex function $\Gamma_{\gamma}$ in nonadiabatic regime. Wavy lines represents the electron-phonon interaction, dashed ones the electron-electron Coulomb repulsion.

tex diagrams [69], while solid lines are the data for the fully vertex corrected theory [63]. For this latter case we show the results for different values of $Q_{c}$.

The first main results of Fig. 16 is that the inclusion of the electron-phonon coupling, in the nonadiabatic regime, yields a sensible reduction of $\chi$ with respect to the pure electronic spin susceptibility $\chi^{e e}$. As expected this effect vanishes as $\lambda \rightarrow 0$ (right panel) or $\omega_{0} / E_{F} \rightarrow 0$ ( left panel). Note that both the noncrossing approximation and the vertex corrected theory yield a similar reduction. This is quite different

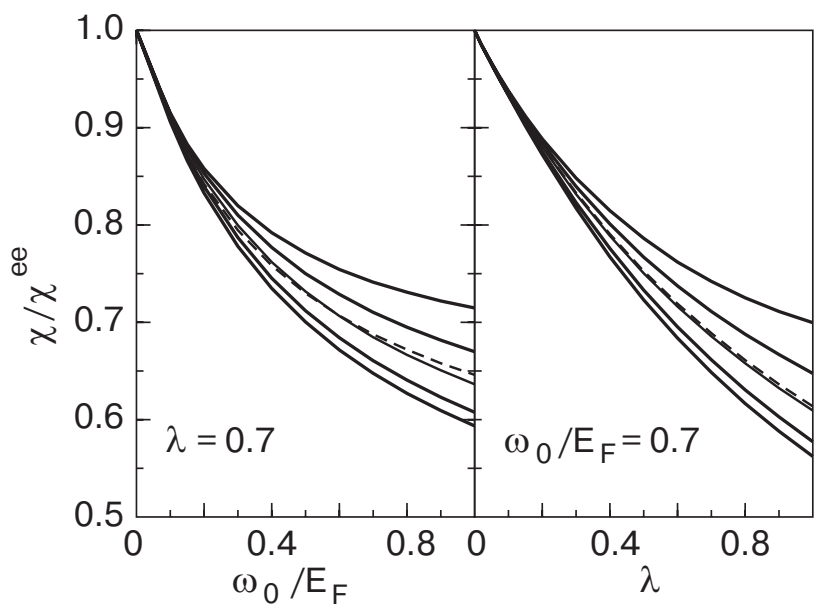

Fig. 16. Spin susceptibility as function of the adiabatic parameter $\omega_{0} / E_{F}$ and of the electron-phonon coupling $\lambda$. The total Pauli spin susceptibility $\chi$ is normalized with respect to the purely electronic one $\chi^{e e}$ with a Stoner factor $N(0) I=0.4$. Dashed lines represent the spin susceptibility in the noncrossing approximation, solid lines are the nonadiabatic theory with vertex diagram (from lower to upper line: $Q_{c}=0.1,0.3,0.5,0.7,0.9$ ). 


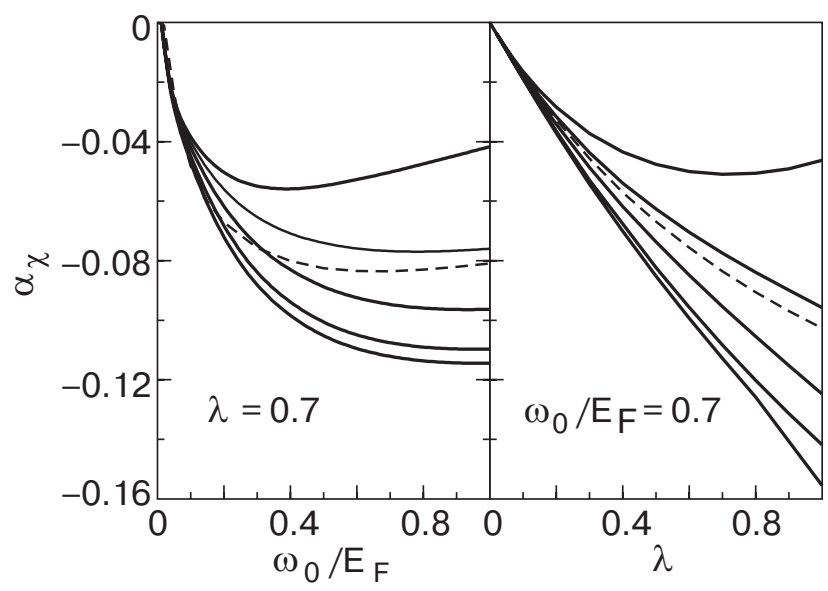

Fig. 17. Isotope effect on the spin susceptibility as a function of $\omega_{0} / E_{F}(\lambda=0.7, N(0) I=0.4)$ and as a function of $\lambda$ $\left(\omega_{0} / E_{F}=0.7, N(0) I=0.4\right)$. Solid lines and dashed lines as in the previous figure.

from the situation encountered in the superconducting pairing channel, where the effect of the vertex diagrams is much stronger and highly dependent on $Q_{c}$ $[38,39]$.

A more clear signature of the nonadiabatic effects is in addition provided by the isotope dependence of the Pauli spin susceptibility. In Fig. 17 we report the numerical calculations of the isotope coefficient $\alpha_{\chi}=-d \log \chi / d \log M_{\text {at }}=(1 / 2) d \log \chi / d \log \omega_{0} \quad$ as function of the adiabatic ratio ${ }^{\circ} 0 / E_{F}$ and of $\lambda$ $[63,69]$. A finite and negative isotope effect is thus predicted with a strong dependence on $Q_{c}$ and $\lambda$ in the intermediate nonadiabatic regime. The observation of the isotope effect, which would be absent in metals fulfilling the Migdal-Eliashberg framework, could be therefore an additional and stringent evidence for a nonadiabatic electron-phonon coupling.

\subsection{Photoemission and real axis analysis}

In Secs. 3.1-3.3 we have briefly discussed the nonadiabatic effects on some one- and two-particle normal state properties. This analysis was simplified by the fact that all these quantities (the effective mass $m^{*}$, the critical temperature $T_{c}$, the zero temperature superconducting gap $\Delta$, the Pauli spin susceptibility $\left.\chi_{P}\right)$ are «thermodynamical» properties, meaning that they are static quantities which can be determined within the context of the imaginary frequency Matsubara space. On the other hand, the anomalous electron-phonon and isotope effects reported in cuprates by the photoemission spectroscopy give rise to a deep interest about the role of nonadiabaticity on frequency-dependent spectroscopic quantities. This task is however made quite difficult by the need of an analytical continuation from imaginary to real axis frequencies, which in the mean-field-like ME theory results to be relatively simple but which in the nonadiabatic vertex corrected context becomes very hard. In this section we summarize thus only nonadiabatic anomalies which arise from finite band effects neglecting for the moment the explicit inclusion of the vertex diagrams. We shall see however that the photoemission phenomenology presents already at this level many interesting features. In particular we see that the following fundamental el-ph properties $[27,70]$ are no longer valid when $E_{F} \sim \omega_{0}$ :

i) the el-ph self-energy $\Sigma(\omega)$ does not renormalize the electronic dispersion for 10 much larger than the phonon energy scale $\omega_{0}$;

ii) impurity scattering affects only the imaginary part of the self-energy but not the real part, and hence not the electronic dispersion;

iii) different channels of electron scattering (phonons, impurities, ...) sum linearly in the self-energy.

The small Fermi energy effects on the real axis due to the finite bandwidth can be conveniently dealt with by means a proper generalization of the Marsiglio-Schossmann-Carbotte technique [71], which in finite bandwidth systems and in the presence of impurity scattering reads [72]:

$$
\begin{aligned}
& \Sigma\left(i \omega_{n}\right)=-2 i T \sum_{m} \lambda\left(i \omega_{n}-i \omega_{m}\right) \eta\left(\omega_{m}\right)-2 i \gamma \eta\left(\omega_{n}\right) \\
& \Sigma^{\prime}(\omega)=2 T \sum_{m} \lambda^{\prime}\left(\omega, \omega_{m}\right) \eta\left(\omega_{m}\right)-\int_{-\infty}^{\infty} d \Omega \alpha^{2} F(\Omega) \times \\
& \times[N(\Omega)+f(\Omega-\omega)] \eta^{\prime}(\omega-\Omega)-\gamma \eta^{\prime}(\omega) \\
& \Sigma^{\prime \prime}(\omega)=-\int_{-\infty}^{\infty} d \Omega \alpha^{2} F(\Omega)[N(\Omega)+f(\Omega-\omega)] \times \\
& \times \eta^{\prime \prime}(\omega-\Omega)-\gamma \eta^{\prime \prime}(\omega),
\end{aligned}
$$

where $N(x)$ and $f(x)$ are the Bose and Fermi distribution functions, respectively, $\alpha^{2} F(\Omega)$ is the el-ph Eliashberg function, $\gamma$ is the impurity scattering rate. Moreover

$$
\lambda(z)=\int_{-\infty}^{\infty} d \Omega \alpha^{2} F(\Omega) /[\Omega-z]
$$

$(z$ complex number $), \lambda^{\prime}\left(\omega, \omega_{m}\right)=\operatorname{Im} \lambda\left(\omega-i \omega_{m}\right)$, and

$$
\begin{gathered}
\eta\left(\omega_{m}\right)=\arctan \left[\frac{E_{F}}{\omega_{m} Z(\omega m)}\right], \\
\eta^{\prime}(\omega)=\frac{1}{2} \ln \left[\frac{\left[E_{F}-\omega Z^{\prime}(\omega)\right]^{2}+\left[\omega Z^{\prime \prime}(\omega)\right]^{2}}{\left[E_{F}+\omega Z^{\prime}(\omega)\right]^{2}+\left[\omega Z^{\prime \prime}(\omega)\right]^{2}}\right], \\
\eta^{\prime \prime}(\omega)=\arctan \left[\frac{E_{F}-\omega Z^{\prime}(\omega)}{\omega Z^{\prime \prime}(\omega)}\right]+\arctan \left[\frac{E_{F}+\omega Z^{\prime}(\omega)}{\omega Z^{\prime \prime}(\omega)}\right],
\end{gathered}
$$


where $Z(z)=1-\Sigma(z) / z$.

In Fig. 18, $a$ we plot the real and imaginary part of the self-energy for an el-ph Einstein model with $E_{F}=4 \omega_{0}$ and $\lambda=1$ in the presence of impurity scattering. Let us first the nonadiabatic self-energy for $E_{F}=4 \oplus_{0}$ with the ME one $\left(E_{F}=\infty\right)$ in the $\gamma=0$ limit (dashed line). Note that in this latter case the real
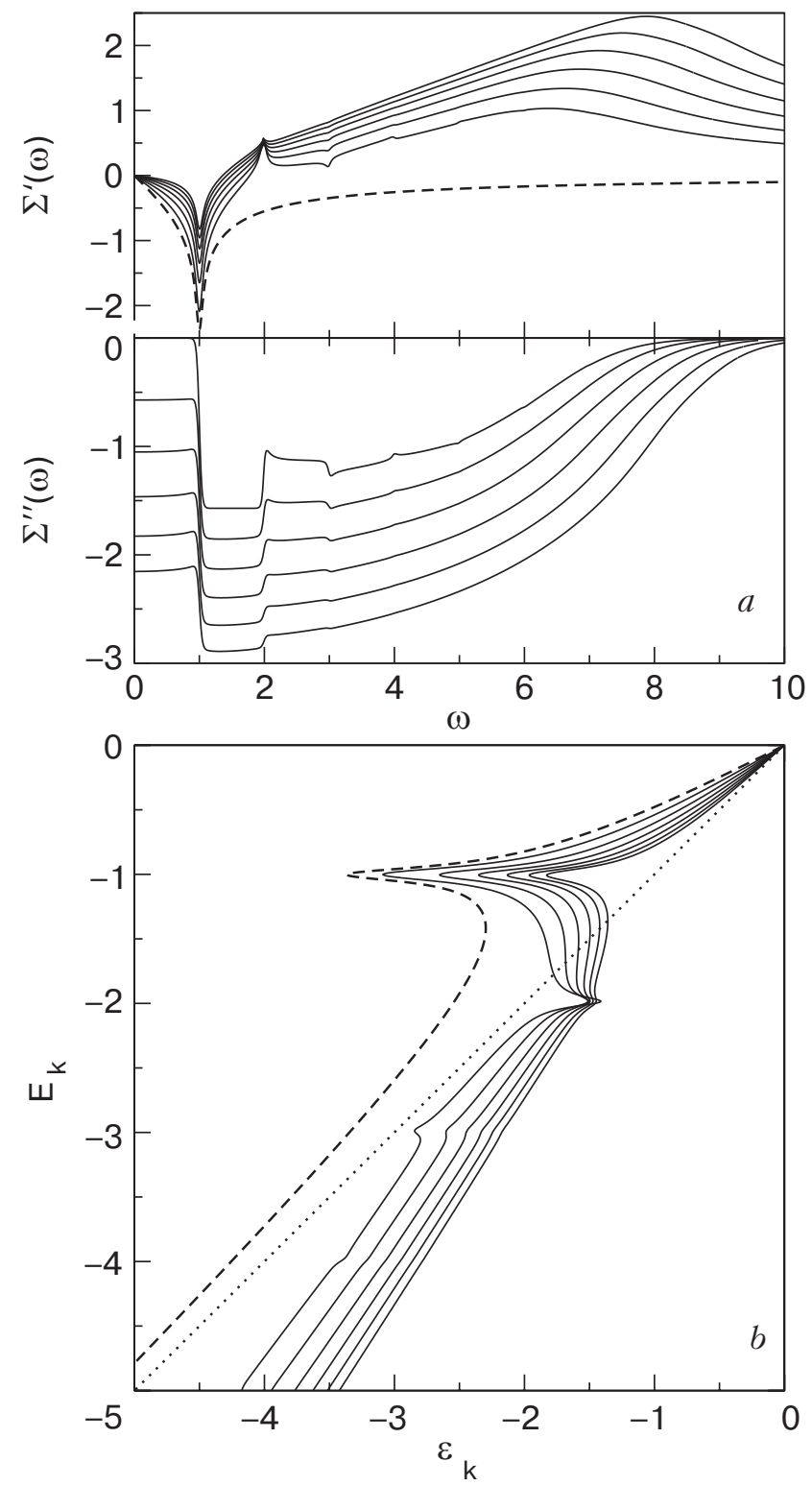

Fig. 18. Panel $a$ : Real and imaginary part of $\Sigma$ for a Einstein phonon mode with $\lambda=1$ and $E_{F}=4 \omega_{0}$ in the presence of impurity scattering. Solid lines corresponds to (upper panel: from bottom to the top; lower panel: from top to the bottom): $\gamma / \omega_{0}=0,0.2,0.4, \ldots, 1.0$ where $\gamma$ is the impurity scattering rate. Energy quantities are expressed in units of $\omega_{0}$. The dashed line in the upper panel is the real part of the self-energy in the adiabatic infinite bandwidth limit $E_{F}=\infty$. Panel $b$ : renormalized electron dispersion corresponding (from left to the right) to panel $a$. The dashed line represents the adiabatic limit. part of the self-energy is always negative implying that the effective electronic band $E_{\mathbf{k}}$ is always less steep than the bare one $\varepsilon_{\mathbf{k}}: E_{\mathbf{k}} \leq \varepsilon_{\mathbf{k}}$ for any energy. In addition the low-energy part of $\Sigma^{\prime}(\omega)$ is just $\Sigma^{\prime}(\omega) \sim \lambda(0$, which gives the well-known renormalization of the electronic dispersion $E_{\mathbf{k}}=\varepsilon_{\mathbf{k}} /(1+\lambda)$ close to the Fermi level $[27,70]$, while for $\omega>\omega_{0}$ the $\Sigma^{\prime}(\omega)$ goes rapidly to zero implying that no significant renormalization effect is expected. Note also that the magnitude of $\Sigma^{\prime \prime}(\omega)$ is a monotonously increasing function with $\omega$ and saturates for $\omega \geq \omega_{0}$.

The presence of a Fermi energy of the same order of the phonon frequencies gives rise to a number of qualitative new features. Most important here we would like to signalize [72]: i) $\Sigma^{\prime \prime}(\omega)$ is no longer a monotonous function of $\omega$, but when $\omega$ becomes roughly $\omega \gtrsim E_{F}$ the imaginary part of the self-energy starts to decrease in modulus and it goes quite rapidly to zero. This is easily understandable in small Fermi energy systems if one considers that for $\omega \gg E_{F}$ there are no electronic states into which an electron with energy 1 could decay within an energy window $\sim \omega_{0}$. Another interesting feature is indeed the large positive hump of the real part of the self-energy which occurs in correspondence of the drop of the imaginary part and it scales with $E_{F}$. In particular we note that, in contrast with the case $E_{F}=\infty$, for finite $E_{F}$ the real part of the self-energy $\Sigma^{\prime}(\omega)$ becomes positive in a large range of energy for $₫ \gtrsim 2 \Theta_{0}$. Finally, by looking at the effect of the impurity scattering, we note that the presence of two sources (impurities and phonons) of scattering does not sum linearly neither in $\Sigma^{\prime}(\omega)$ neither in $\Sigma^{\prime \prime}(\omega)$. These anomalous features have an important impact on the renormalized electronic dispersion obtained by $E_{\mathbf{k}}-\varepsilon_{\mathbf{k}}-\Sigma^{\prime}\left(E_{\mathbf{k}}\right)=0$ which corresponds in ARPES measurements to the dispersion inferred by the momentum distribution curves (MDC). As shown in Fig. 18,b the positive part of $\Sigma^{\prime}(\omega)$ implies an «anti-renormalization» of the electron band, namely $E_{\mathbf{k}}>\varepsilon_{\mathbf{k}}$. This new feature extends up to an energy scale which does not depend on $\omega_{0}$ but only on $E_{F}$, while its magnitude depends on el-ph parameters as $\lambda$ or $E_{F}$ itself. In such a situation the high-energy part $E_{\mathbf{k}}>\omega_{0}$ of the experimental electronic dispersion $[16,73]$ does not represent anymore the bare band $\varepsilon_{\mathbf{k}}$ but it expected to show a steeper behavior than $\varepsilon_{\mathbf{k}}$. In addition the dependence of the electronic dispersion on the impurity scattering shows that the high-energy part of $E_{\mathbf{k}}$ is still highly dependent on the microscopic details not only of the electron-phonon scattering but also of any other source of electronic scattering, as for instance here impurities. This consideration could account for the strong dependence of the high-energy part of the ARPES data on the hole doping $\delta$ in 
cuprates, where the electron-electron interaction is expected to be strongly dependent on $\delta$ [73].

\section{Nonadiabatic superconductivity in fullerides and cuprates}

In the previous section we have introduced the general equations of the superconducting and normal state theories of nonadiabatic systems. A number of different properties have been explicitly evaluated in simple models in order to point out the anomalous nonadiabatic features arising from the opening of new electron-phonon interaction channels related to the vertex diagrams. While the nonadiabatic theory above discussed would apply in full generality in a wide variety of nonadiabatic systems, specific features of different materials would depend of course on specific microscopical and materials-science details. In the course of our discussion we have already linked the predictions of the nonadiabatic theory with some unconventional experimental findings in cuprates and fullerides. In this last part we would like thus to address in more details the specific nonadiabatic superconducting properties of these compounds.

In regards with the nonadiabatic features, fullerides represent a very important test since, due to the extremely high frequencies of the intra-molecular phonon spectrum and to the weak inter-molecular electron hopping [74-76], the nonadiabatic ratio ${ }^{(1} \mathrm{ph} / E_{F}$ is significantly large, ${ }^{\left({ }^{0} \mathrm{ph}\right.} / E_{F} \simeq 0.4-0.8$ $[74,75]$. On the other hand, in cuprates the two-dimensional character of the electronic band and the closeness of the Fermi level to a logarithmic Van Hove singularity [77] permit to investigate additional nonadiabatic effects triggered by the flatness of the electronic bands near the Van Hove saddle point. In both these materials the large local Hubbard repulsion $U$ compared to the small Fermi energy $E_{F}$ gives rise to strong correlation effects.

\subsection{Correlation effects on the electron-phonon scattering}

The interplay between the electronic correlation and the electron-phonon interaction plays a fundamental role within the context of the nonadiabatic theory of the electron-phonon interaction. To better understand this issue we remind that the nonadiabatic effects, especially on the superconducting pairing, depend significantly on the sign of the vertex processes. In particular we have shown that a predominance of forward scattering with small momentum $|\mathbf{q}|$ would lead to an enhancement of the effective electronphonon coupling by selecting positive regions of the vertex function [37-39]. From the physical point of view this situation has been argued in literature to be naturally realized in systems with a strong degree of electronic correlation [52-57].

The effects of strong electronic correlations on the one-particle properties has been already studied in extensive way in the literature [78-81]. Common wisdom describes the evolution from a free-like system to a correlated one in term of coherent and incoherent parts of the Green's function $G(\mathbf{k}, \omega)$. In the context of a Fermi liquid picture the coherent part of $G$ can be written as [78-80,82]:

$$
G_{\text {coh }}(\mathbf{k}, \omega)=\frac{Z}{\omega-Z \varepsilon(\mathbf{k})},
$$

where the reduced quasi-particle weight $Z$ which renormalizes also the quasi-particle dispersion is due to the electronic correlation and it depends on the microscopic parameters of the system. In the context of Hubbard model for instance $Z=Z(U, n)$, where $U$ is the on-site Hubbard repulsion and $n$ is the electron density per site. One-particle correlation effects can be thus parametrized in terms of $Z$ where $Z \sim 1$ represents a weakly correlated system and $Z \rightarrow 0$ a strong correlation regime. For $Z=0$ a metal-insulator transition is expected where quasi-particle weight vanishes [78-82].

Concerning the interplay between the electron-electron correlation and the electron-phonon interaction a crucial interest is paid to the two-particle response functions. In particular, since phonons are directly coupled to the electronic charge, a primary role is played by the charge density response. Work in this direction has been mainly based on analytic tools, as slave-boson techniques $[52,57,83-86]$ or Hubbard $X$-operator formalism [53-55], recently supported by quantum Monte Carlo calculations [56]. Object of interest has been the effect of the electronic correlation on the phonon mediated electron-electron interaction $\lambda\left(\mathbf{q}, \omega_{q}\right)$ or, equivalently, on the electron-phonon matrix element $g(\mathbf{q})$ which, in the absence of correlation, can be assumed to be shapeless $g^{0}(\mathbf{q}) \simeq g$. The two quantities are simply connected by $\lambda\left(\mathbf{q}, \oplus_{q}\right)=$ $=|g(\mathbf{q})|^{2} D\left(\omega_{q}\right)$, where $D\left(\omega_{q}\right)$ is the phonon propagator.

The evolution of $|g(\mathbf{q})|^{2}$ on the degree of electronic correlation has been widely studied. In Refs. $52-57$ it was shown that a prominent peak arises for small $|\mathbf{q}|$ scattering by increasing the electronic correlation while large $|\mathbf{q}|$ momenta are strongly suppressed. In the strong correlation regime this peaked structure in $|\mathbf{q}|$ can be in good approximation described by a Lorentzian: 


$$
|g(\mathbf{q})|^{2} \propto g^{2} \frac{1}{|\mathbf{q}|^{2}+\xi^{-2}},
$$

where $\xi^{-1}$ represents the inverse correlation length which can be identified with the cut-off parameter $q_{c}$ introduced in Sec. 2. The small momenta selection becomes more and more pronounced by approaching the metal-insulator transition and for $Z \rightarrow 0, q_{c} \rightarrow 0$ [52-57].

The concept of correlation length can be useful to get a physical understanding of the small $|\mathbf{q}|$ selection. Let us consider an electron on the site $i$ in a uncorrelated system. In good approximation it interacts with the other electrons only through their mean density. The space positions of the single electrons are thus independent each others (Fig. 19, $a$ ) and any spatial charge modulation with wave vector $\mathbf{q}$ probes the same equal electronic response.

Things are different in correlated systems. In this case indeed the position of one electron at the site $i$ is correlated with the position of the other electrons within a radius $\xi$ (Fig. 19,b) [79]. The internal dynamics within a size $\xi$ is thus frozen out and charge modulations with wavelength $l<\xi$ are prevented. In the momentum space this is reflected in a predominance of small momenta $|\mathbf{q}|<\xi^{-1}$ and in a suppression of the large ones $|\mathbf{q}|>\xi^{-1}$.

We can now understand the empirical relation between the high critical temperature superconductivity and the strong electronic correlation on the basis of the nonadiabatic scenario. Nonadiabatic effects and a strong electronic correlation are natural by-products of small Fermi energy systems when $E_{F}$ becomes comparable with both the phonon and the Hubbard energy scales. The first feature (nonadiabaticity) gives rise to new interaction channels which can enhance or suppress the electron-phonon coupling depending on $\mathrm{mi}^{-}$ croscopic details [37-39]; the second one (electronic correlation, small $|\mathbf{q}|$ momenta) will select the positive

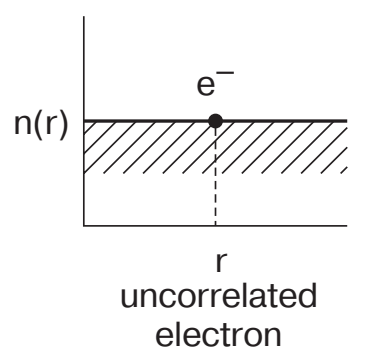

$a$

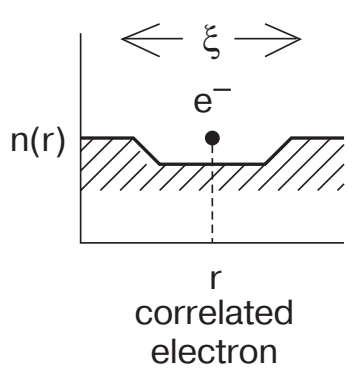

$b$
Fig. 19. Schematic picture of uncorrelated $(a)$ and correlated (b) electrons. Correlated systems are characterized by the correlation hole (depicted in panel $b$ ) which surrounds each electron and prevent charge fluctuations with wavelength less than $\xi$. part of the vertex processes and as a consequence it will favor the resulting electron-phonon coupling enhancement due to the nonadiabatic effects. According this picture strongly correlated systems appear as natural good candidate for high- $T_{C}$ superconductivity within the context of the nonadiabatic theory [39]. The constructive interplay between nonadiabatic electron-phonon interaction and electronic correlation, and the role of the modulation of the small-q scattering as function of the correlation degree, are pointed out in the most remarkable way in the fullerene compounds and in cuprates.

\subsection{Fullerenes}

Superconductivity in fullerenes is commonly associated with $\mathrm{A}_{3} \mathrm{C}_{60}$ compounds [74,75]. Pristine $\mathrm{C}_{60}$ is a band insulator where the highest molecular orbitals $h_{u}$ are completely filled [74,75]. Chemical intercalation with alkali atoms, which are completely ionized, provides additional charges and permits to dope these systems. Band theory would thus predict a metallic character up to $n=6$ electrons for buckyball when the lowest unoccupied molecular orbitals $t_{1 u}$ are expected to be completely filled. The actual phase diagram vs. $n$ is however quite more complex, and a narrow metallic regime is found only close to $n=3$ in $\mathrm{A}_{3} \mathrm{C}_{60}$ compounds [87]. Present understanding of this anomalous phase diagram is not at all exhaustive and certainly it needs the proper inclusion of the electronic correlation and of anomalous lattice features. It is interesting to note however that superconductivity appears only in the metallic regime. This observation suggests that the metallic character is a fundamental requirement for superconductivity in these systems.

In spite of many characteristic properties of $\mathrm{C}_{60}$ compounds which make them unlikely candidates for high- $T_{C}$ superconductivity in the Migdal-Eliashberg framework (strong electron-electron repulsion, significant electronic correlation, low-carrier density), superconductivity in $\mathrm{A}_{3} \mathrm{C}_{60}$ has been often regarded as conventional [88]. The high critical temperatures $\left(T_{c} \simeq 33 \mathrm{~K}\right.$ in $\mathrm{RbCs}_{2} \mathrm{C}_{60}, T_{c} \simeq 40 \mathrm{~K}$ in $\mathrm{Cs}_{3} \mathrm{C}_{60}$ under pressure) are thus attributed to extremely favorable microscopic values: a particularly strong electronphonon coupling $\lambda \sim 1$ (LDA calculations estimate $\lambda \simeq 0.5-1)$ and high-energy phonon frequencies ${ }^{(1)} \mathrm{ph} \sim 1000-1500 \mathrm{~K}$ [89-91]. These features should thus compensate the relatively small density of states and the strong electron-electron Coulomb repulsion $\mu^{*}=0.4[74,92,93]$. The Jahn-Teller nature of the electron-phonon interaction is suggested in addition to further reduce the effect of the Coulomb repulsion [94]. In this situation the critical temperatures of the 
$\mathrm{C}_{60}$ compounds are close to maximum values theoretically achievable.

On a microscopic ground the standard Migdal-Eliashberg theory is however intrinsically inconsistent with respect to the adiabatic problem. $\mathrm{C}_{60}$ compounds are indeed characterized by a set of very narrow bands with typical $E_{F} \simeq 0.25 \mathrm{eV}$, for both electron and hole doping, whereas phonon mode energies range up to $\omega_{\mathrm{ph}} \simeq 0.2 \mathrm{eV}[95,96]$. The breakdown of the nonadiabatic hypothesis is shown in the most remarkable way in Fig. 20 where the «adiabatic phase diagram» $\left(\omega_{\mathrm{ph}} / E_{F}\right.$ vs. $\left.\lambda\right)$ of fullerenes, obtained by different numerical calculations, is drawn. In this situation the nonadiabatic theory outlined in the previous section is the unavoidably starting point of any realistic description of superconductivity in these materials. In addition we can expect that the interplay between the strong electronic correlation and the nonadiabatic electron-phonon coupling would enhance the superconducting pairing. A significant indication about the relevance of a nonadiabatic effects is given by the observed reduction of $T_{C}$ upon induced disorder [99]. This feature, as we have previously seen, can be considered one of the hallmarks of a nonadiabatic pairing in $s$-wave superconductors [62].

To illustrate the role of the opening of nonadiabatic channels in fullerenes, the concrete example of $\mathrm{Rb}_{3} \mathrm{C}_{60}$ can be useful since for this compound best experimental data are available. Recent measurements have indeed determined with the highest degree of accuracy the carbon isotope coefficient on $T_{c}$,

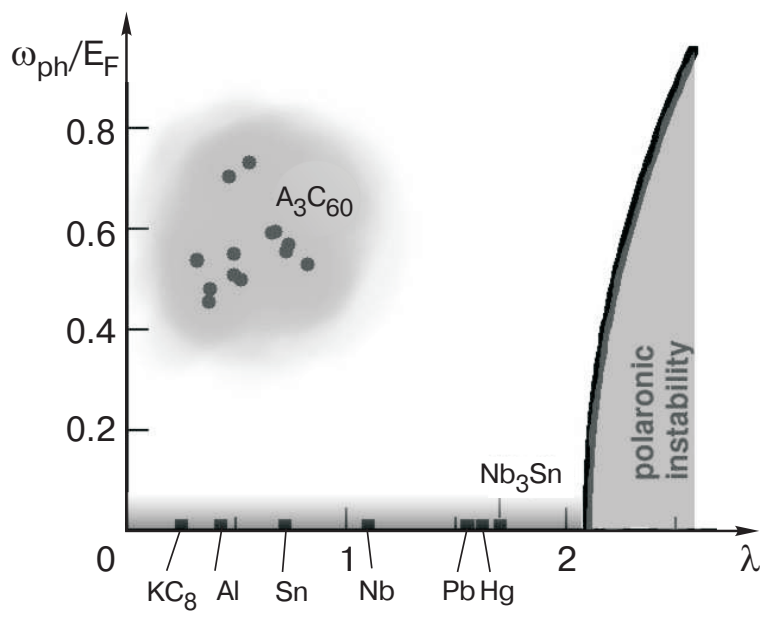

Fig. 20. Phase diagram of conventional superconductors compared with the fullerene compounds in the space defined by the electron-phonon coupling $\lambda$ and the adiabatic parameter ${ }^{0}{ }_{\mathrm{ph}} / E_{F}$. Data for the $\mathrm{A}_{3} \mathrm{C}_{60}$ family where taken from DFT, tight-binding, and ab initio calculations [89-91,97,98] by using standard values for the density of states $N\left(E_{F}\right)=10$ states $/\left(\mathrm{eV}\right.$-spin- $\left.\mathrm{C}_{60}\right)$ and for the Fermi energy $E_{F}=0.25 \mathrm{eV}$.
$\alpha_{T_{c}}=0.21$ [100], which, together with the large critical temperature $T_{c} \simeq 30 \mathrm{~K}$, can be used of basis of analysis to test both Migdal-Eliashberg and the nonadiabatic theories. In Fig. 21 we show the numerical solutions of both the adiabatic Migdal-Eliashberg and the nonadiabatic theory constrained to reproduce the experimental values $T_{c}=30 \mathrm{~K}$ and $\alpha=0.21$ with an Einstein phonon $\omega^{\circ} \mathrm{ph}$. For given values of $\omega^{\mathrm{p} h}$ lying in the physical range of the fullerene phonon spectrum $\omega^{0} \mathrm{ph}<2300 \mathrm{~K}$ a extremely large electronphonon coupling $\lambda \sim 1-4$ is required in ME theory (filled squares), in contrast with local density approximation results which find $\lambda<1$. On the other hand, the same experimental data are fitted in the nonadiabatic theory (open triangles) with much more reasonable values of $\lambda$ in good agreement with the theoretical calculations [95].

We would like to stress that, within the context of the nonadiabatic theory of superconductivity, the high values of the critical temperatures in fullerides are not related to some particularly strong electron-phonon coupling rather more to the onset of higher order diagrams scaling with $\omega_{\mathrm{ph}} / E_{F}$. This perspective has interesting consequences in regards with the optimization of the superconducting properties in these and in other nonadiabatic superconductors. In particular, since the electron-phonon coupling is expected to be in the weak-intermediate regime $\lambda \sim 0.5-1$, the fullerenes are likely to be far from lattice instabilities, which are mostly due to the closeness of the Mott-Hubbard metal-insulator transi-

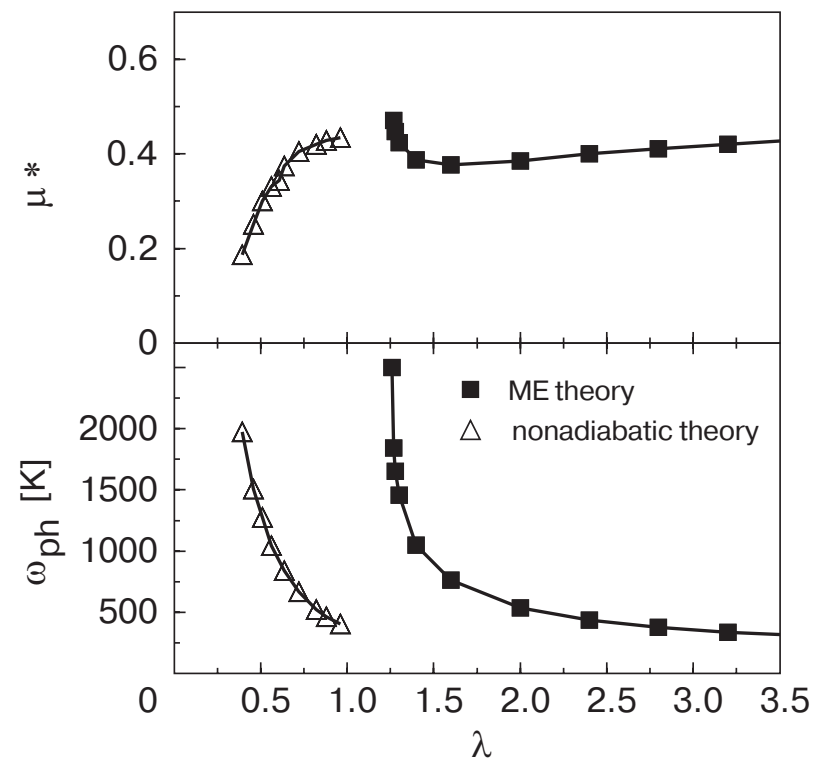

Fig. 21. Phonon frequency ${ }^{1} \mathrm{ph}$ (lower panel) and Coulomb pseudopotential $\mu^{*}$ (upper panel) as functions of the electron-phonon coupling $\lambda$. Both the ME (filled squares) and the nonadiabatic (open triangles) equations have been solved in order to reproduce $T_{C}=30 \mathrm{~K}$ and $\alpha=0.21$. 
tion, so that $T_{C}$ could reasonably be further increased in fullerides upon increasing the electron-phonon coupling itself.

A widely used way to control the electron-phonon coupling in fullerides is by tuning the lattice spacing a. This is usually done in the $\mathrm{A}_{3} \mathrm{C}_{60}$ by varying the alkali atoms A with different ionic sizes [74]. Since the phonon and electron-phonon properties are mainly ruled by intra-molecular properties, the main effect of the lattice spacing is of tuning electronic quantities like the density of states $N(0)$ and the Fermi energy $E_{F}$. Larger lattice parameters $a$ correspond thus to high density of states and to higher $T_{c}$ 's. Another convenient way to obtain large lattice spacing in fullerides is by means of ammonia intercalation. Although at the present ammonia intercalation in $\left(\mathrm{NH}_{3}\right)_{x} \mathrm{~A}_{3} \mathrm{C}_{60}(x \lesssim 1)$ is usually found to suppress superconductivity, it is important to note that the reduction of $T_{c}$ is always accompanied by the reduction of the symmetry of the system, global (long-range antiferromagnetism, lattice distorsion) or local one (crystal field splitting) [101]. According our view such reductions of symmetry are more likely to be related to the electron-electron correlations than to the electron-phonon coupling. In this perspective, we suggest that the electron-phonon coupling and thus the critical temperature could be significantly further increased in ammonia intercalated compounds once these reductions of symmetry are prevented. The high critical temperature $T_{c} \simeq 30 \mathrm{~K}$ of $\left(\mathrm{NH}_{3}\right)_{4} \mathrm{~A}_{3} \mathrm{C}_{60}$ [102], where the full electron and lattice symmetries of the $\mathrm{A}_{3} \mathrm{C}_{60}$ are restored, seems to confirm this picture.

\subsection{Copper oxides}

In comparison with the $\mathrm{C}_{60}$ compounds the cuprate family presents a much more complex phenomenology as it is testified by the richness of anomalous features in the $T$ vs. $\delta$ phase diagram, schematically depicted in Fig. 22. Undoubtedly the revealing of different phases in cuprates is made easier than in fullerenes by the fact that the electronic filling in copper oxides can be tuned in a continuous way by stoichiometric doping, whereas $\mathrm{C}_{60}$ compounds become rapidly insulators as soon as the electron concentration per buckyball moves away from $x \simeq 3$ (half-filling). In addition, although correlation effects are certainly important in fullerenes, the $\mathrm{A}_{3} \mathrm{C}_{60}$ compounds lie on the metallic side of the Brinkmann - Rice transition while cuprates at half-filling are Mott insulator with long range antiferromagnetic order. The appearing of a variety of exotic phases close at half-filling is not surprising since in this regime the quasi-particle kinetic energy of the electrons is highly suppressed so that it can be

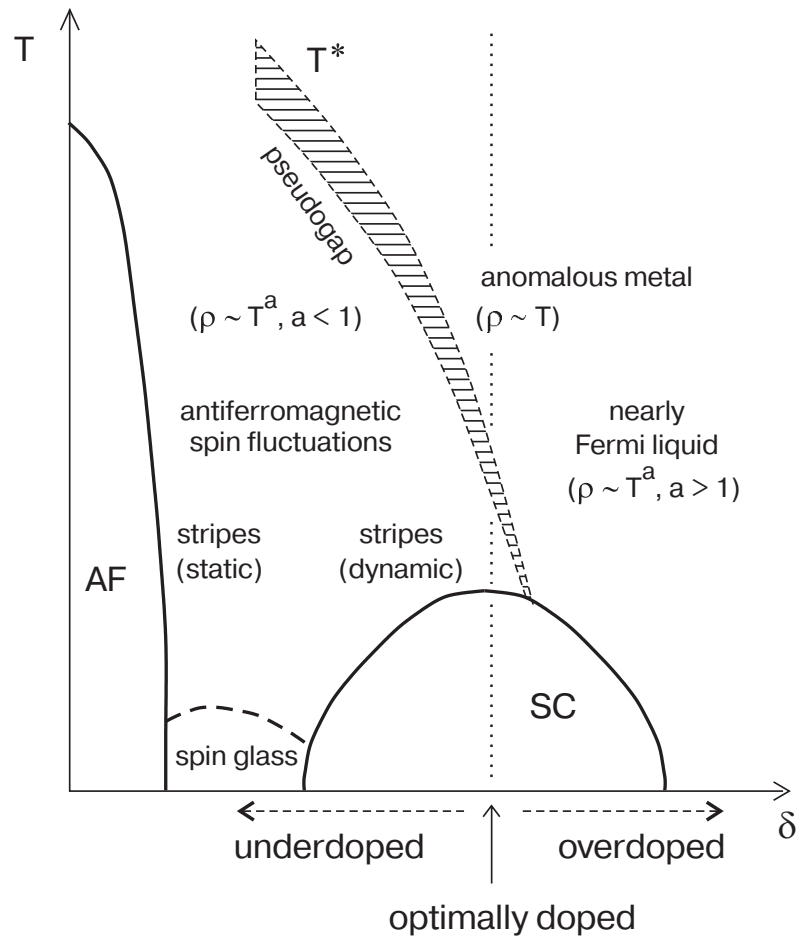

Fig. 22. Schematic phase diagram of the copper oxides compounds in the temperature vs. doping space.

easily overwhelmed by different long-range or shortrange orderings. At the same time, the smallness of the kinetic energy scale, parametrized by the Fermi energy $E_{F}$, gives rise unavoidably also to nonadiabatic effects when compared with the phonon energy scale.

As a general rule, the «bell-shape» profile of the superconducting phase as function of the hole doping is commonly regarded in a twofold way from the scientific community [103]. According the first point of view, the onset of some long- or short-range ordering (AF fluctuations, stripes, spin glasses, pseudogap, CDW quantum critical point) is the active principle for the high- $T_{C}$ superconductivity [104-114]. Along this perspective the disappearing of these features and the restoring of normal metal properties in the overdoping region leads to a reduction of $T_{c}$. The above scenario is reversed according the second point of view which regards the suppression of $T_{C}$ in the underdoping regime as arising from the competition of the superconducting phase with other different kinds of ordering [115-118]. In this scenario more emphasis is thus paid to the overdoped region where other actors detrimental for the superconductivity are absent and it should be easier to identify the mechanism underlying the superconducting pairing.

The nonadiabatic theory of superconductivity permits to understand in a very natural way the bellshape of the superconducting phase diagram [119]. The active principle in this context is the onset of the 
nonadiabatic channels of interaction. A crucial tuning role however is also played by the electronic correlation which promotes small $\mathbf{q}$ scattering and selects the positive (attractive) parts of the vertex diagrams. Moving from the overdoped to the underdoped region we expect thus the electronic correlation to be higher, the small $\mathbf{q}$ selection more effective and the effective superconducting pairing stronger. This trend, which is essentially based on the doping dependence of the particle-particle Cooper interaction, has however to compete with the reduction of the one-particle spectral weight, which roughly scales with $\delta$ in the low doping regime $[78,79,82]$. The phase diagram resulting from the competition of these two kinds of effects can be conveniently discussed in terms of the linearized superconducting kernel in the nonadiabatic regime [119], Eq. (37), which can be rewritten in a simplified way as:

$$
\varphi_{n} \simeq T_{c} \sum_{m} Z \lambda[1+2 Z \lambda P+Z \lambda C] \frac{\varphi_{m}}{W_{m}} \arctan \left(\frac{E_{F}}{W_{m}}\right),
$$

where the prefactor $Z$ in front of the superconducting kernel and of the vertex and cross diagrams arises from the quasi-particle spectral weight reduction according Eq. (44), and where we neglect for sake of simplicity the frequency dependence of the nonadiabatic contributions $P, C$. In this way, we can roughly see the total electron-phonon coupling as the product of two terms: an effective electron-phonon coupling of ME theory renormalized by the electronic correlation, $\lambda^{M E}$, and the enhancement due to nonadiabatic vertex and cross (vc) diagrams $\gamma^{v c}$ :

$$
\begin{aligned}
& \lambda^{\mathrm{eff}}=\lambda^{M E} \gamma^{v c}, \\
& \lambda^{M E}=Z \lambda, \\
& \gamma^{v c}=1+2 Z \lambda P\left(Q_{c}\right)+Z \lambda C\left(Q_{c}\right) .
\end{aligned}
$$

The schematic behavior of these quantities as a function of the hole doping $\delta$ is shown in the upper panel of Fig. 23 [119]. The physics behind the $\delta$-dependence of $\lambda^{M E}$ can be easily related to the loss of spectral weight approaching the metal-insulator transition for $\delta \rightarrow 0$. This effect, which is present also in $\gamma^{v c}$, is however in that case competing with the enhancement of the effective coupling due to $P\left(Q_{c}\right)$ and $C\left(Q_{c}\right)$ which will be maximum and positive close to half-filling (where $Q_{c} \rightarrow 0$ ) and negative at high dopings. The interplay between these two effects will give rise to a maximum of $\gamma^{v c}$, and hence of $\lambda^{\text {eff }}$, somewhere in the small doping region where the competition between the spectral weight loss and the pos-

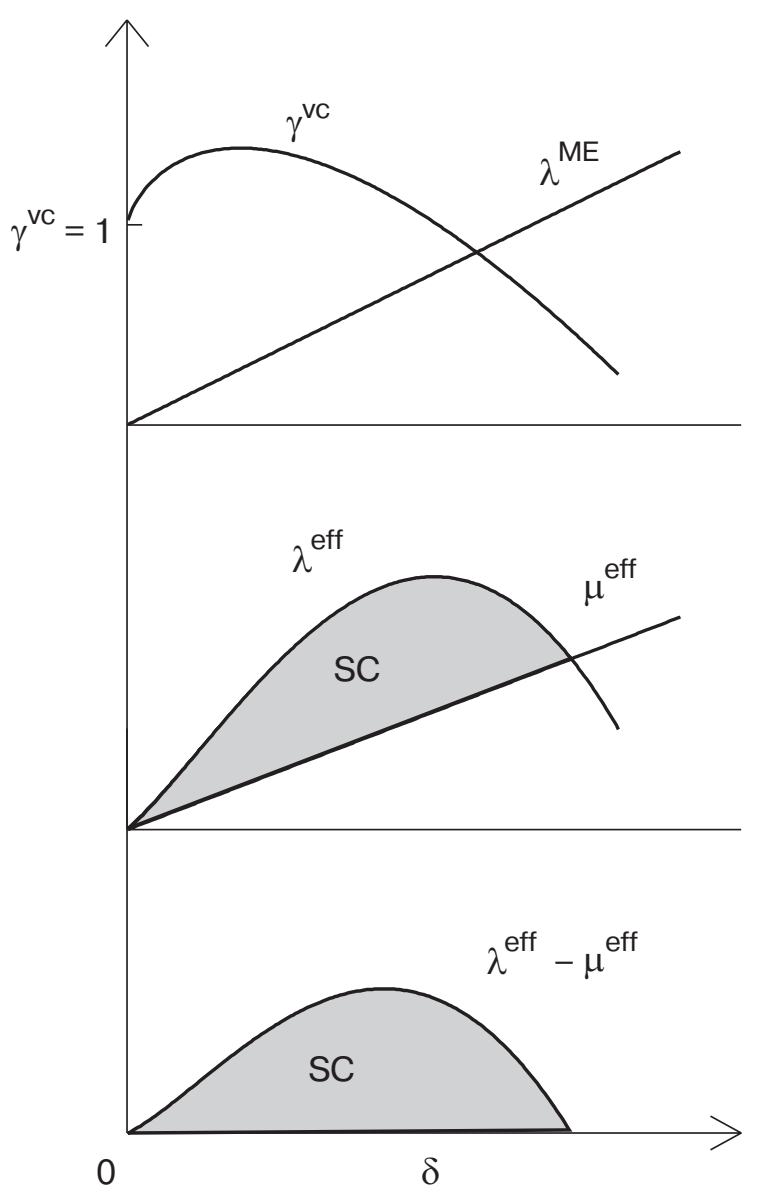

Fig. 23. Graphical sketch of the different contributions to the effective superconducting coupling. Top panel: the coupling function $\lambda^{M E}$ is mainly determined by the coherent spectral weight, and it exhibits a monotonous growing behavior as a function of doping. The vertex factor $\gamma^{\gamma c}$ tends to enhance the effective coupling at low doping and to depress it at high doping. Middle panel: the total effective electron-phonon coupling $\lambda^{\text {eff }}=\lambda^{M E} \gamma^{v c}$ has a maximum at some finite value of $\delta$; when the effective MorelAnderson pseudopotential is subtracted, superconductivity is suppressed at high doping. Lower panel: resulting phase diagram for superconductivity: superconductivity is only possible in a finite region of phase space (gray region), where $\lambda^{\text {eff }}-\mu^{\text {eff }}$ is positive.

itive nonadiabatic effects is stronger (see upper and middle panels in Fig. 23).

We can now also consider the effect of the residual Morel-Anderson-like repulsion $\mu$; first of all, we observe that the reduction of spectral weight will lead to an effective repulsion $\mu^{\text {eff }} \simeq Z \mu$. Superconductivity will be possible only when the net electron-phonon attraction overcomes the repulsion term: $\lambda^{\text {eff }}-\mu^{\text {eff }}>0$ (see lower panel of Fig. 23) [119]. The resulting total coupling is expected to exhibit a bell-shape which is mostly due to the $\delta$-dependence of the nonadiabatic factor $\gamma^{v c}$. It is interesting to note two things. First, in 
the extreme case $\lambda^{M E} \lesssim \mu^{\text {eff }}$, where no superconductivity would be predicted in the whole $\delta$ range by the conventional ME theory, we could expect finite $T_{c}$ in a small $\delta$ region, due to purely nonadiabatic effects $\lambda^{\text {eff }}=\lambda^{M E} \gamma^{v c}>\mu^{\text {eff }}$. Secondly, it is clear that within the ME framework a net attractive interaction in the Cooper channel at a certain doping $\delta$, which corresponds to $\lambda^{M E}>\mu^{\text {eff }}$, would imply a superconducting order also at larger $\delta$ since the two quantities $\lambda^{M E}, \mu^{\text {eff }}$ scale in the same way $\propto Z$; on the other hand, in the nonadiabatic theory superconductivity, $T_{C}$ is expected to be limited to some maximum value of doping, due to the negative contribution of the nonadiabatic diagrams $P$ and $C$ at large $\delta$ (large $Q_{c}$ 's).

We would like to stress that the above phase diagram was drawn keeping in mind a minimal scenario where the only actors were the enhancement of the particle-particle interaction due to the nonadiabatic terms and the reduction of the one particle spectral weight. It is understood on the other hand that an exhaustive description of the different features of the experimental phase diagram, including also the normal state, involves unavoidably the taking into account of a series of other different factors.

Without entering in details, we would like just to mention several features of the cuprates which appear unconventional according the Migdal-Eliashberg theory but which can be naturally accounted for within the nonadiabatic context. We have previously already mentioned the onset of unconventional isotope effects both on $T_{c}$, with isotopic coefficient $\alpha_{T_{C}} \sim 0.2-0.8$ either larger or smaller than the BCS limit, and on the effective electronic mass $m^{*}$, with $\alpha_{m^{*}}<0$. Both these features are indeed experimentally observed in cuprates [6-10]. Another apparently puzzling issue in cuprates regards the symmetry of the superconducting order parameter. While the $d$-wave symmetry seems well assessed in hole doped systems [120], the debate is still open about the symmetry in electron doped materials, where some indications suggest a transition from $d$ - to $s$ - or to other anomalous symmetries as function of doping, maybe also in the hole-doped compounds [121-126]. The $s$-wave symmetry is on the other hand well accepted in fullerenes. In this situation it seems difficult to reconcile the different phenomenology of these different systems with a unique superconducting mechanism. In hole-doped cuprates the $d$-wave symmetry is often discussed to rule out an electron-phonon mechanism. This view is however based on the assumption of an isotropic electron-phonon scattering $\left|g^{2}(\mathbf{q})\right| \simeq g^{2}$, which is significantly violated in correlated systems as discussed in Sec. 4.1. In this situation, the momentum structure of the attractive electron-phonon interaction + the elec- tron-electron repulsion can give rise to different symmetry of the superconducting order parameter, from $s$ to $d$-wave, depending on the momentum cut-off $|\mathbf{q}|_{c}$ $[55,127,128]$, so that the electron-phonon interaction results to be a valid candidate for explaining the observation of different symmetries within the context a unique pairing mechanism. As a final remark we would like to mention the report of the linear behavior in temperature of the resistivity in cuprates at optimal doping [129]. This strict linear dependence has also been discussed as an evidence of a nonphononic scattering. However, specific studies show that the linear behavior $\rho(T) \sim T$ can be also naturally explained within the context of an electron-phonon interaction by taking into account the Van Hove singularity effects [130]. Note that the flatness of the electronic band associated with the Van Hove singularity is expected to give rise unavoidably to nonadiabatic effects [131].

\section{Conclusion}

The interest about nonadiabatic effects in high- $T_{C}$ superconductors arises from the experimental observation of a small energy scale associate with the electronic dynamics, characterized by the Fermi energy $E_{F}$. In the high- $T_{c}$ materials the Fermi energy is comparable with the phonon frequency scale ${ }^{\infty} \mathrm{ph}$ $\left(\omega_{\mathrm{ph}} \sim E_{F}\right)$. In this situation the adiabatic assumption of Migdal's theorem $\left(\omega_{\mathrm{ph}} / E_{F} \sim 0\right)$, on the basis of the conventional picture of electron-phonon interaction in metals, breaks down. A novel approach, which takes explicitly into account the nonadiabatic effects in this new regime, is thus unavoidably required.

In the present contribution we have shown how a nonadiabatic theory of the superconductivity and of the normal state can naturally account for the anomalous phenomenology of various high- $T_{c}$ compounds, where we have focused on fullerides and copper oxides. In these compounds, in particular, the origin of the high critical temperature stems out from the interplay between strong electronic correlation and electron-phonon coupling in the new nonadiabatic regime where new channels of electron-phonon scattering, due to the breakdown of Migdal's theorem, are operative. The nonadiabatic theory of superconductivity provides thus an unifying scenario for different kinds of «exotic» superconductors. Within this framework it is also interesting to mention the specific case of $\mathrm{MgB}_{2}$, where the origin of the nonadiabatic effects is slightly different. In this compounds, indeed, band structure calculations predict a Fermi energy $E_{F} \sim 0.4-0.5$ [132-134], to be compared with the 
relevant phonon frequencies $\omega_{\mathrm{ph}} \sim 70-80 \mathrm{meV}$ [133-137]. This simple analysis would thus predict a nonadiabatic ratio $\omega_{\mathrm{ph}} / E_{F} \sim 0.1-0.2$, which would locate $\mathrm{MgB}_{2}$ in the weakly nonadiabatic regime $[138,139]$. A peculiar feature of this system is however that the electron-phonon coupling is mainly concentrated in only one-phonon mode, which is characterized by a very strong deformation potential $I$ [135-137]. In this context new nonadiabatic effects are thus triggered by the quantum lattice fluctuations $[140,141]$ which lead to modifications of the band structure of the same order of the Fermi energy itself $I\left\langle u^{2}\right\rangle^{1 / 2} \sim E_{F}$, where $\left\langle u^{2}\right\rangle^{1 / 2}$ is the root means square of the lattice displacements. Work is at the moment in progress to formalize this new kind of breakdown of the adiabatic assumption. We think that further research on this field would provide, for this class of materials as well as in fullerides and cuprates, new routes for the optimization of the superconducting properties of the existing materials and for the search of new high- $T_{C}$ compounds based on a nonadiabatic type of pairing.

\section{Acknowledgments}

The authors acknowledge fruitful collaborations on this subject with C. Grimaldi, S. Strässler, P. Benedetti, M. Scattoni, P. Paci, M. Botti, L. Boeri, S. Ciuchi and G.B. Bachelet. We also acknowledge financial support from the MIUR projects COFIN03 and FIRB RBAU017S8R.

1. For a review on conventional low- $T_{C}$ metals see: Superconductivity, R.D. Parks (ed.), Dekker, New York (1969).

2. P.W. Anderson and C.C. Yu, in: Highlights of Condensed Matter Theory, Proc. of the Int. School of Physics «E. Fermi», LXXXIX, F. Bassani and M. Tosi (eds.), North-Holland, New York (1985).

3. P.B. Allen and B. Mitrovic, in: Solid State Physics, v. 37, H. Ehrenreich, D. Turnbull, and F. Seitz (eds.), Academic Press, New York (1982).

4. J.G. Bednorz and K.A. Müller, Z. Phys. B64, 189 (1986).

5. L.C. Bourne, M.F. Crommie, A. Zettl, H.C. zur Loye, S.W. Keller, K.J. Leary, A.M. Stacy, K.J. Chang, and M.L. Cohen, Phys. Rev. Lett. 58, 2337 (1987).

6. M.K. Crawford, W.E. Farneth, E.M. McCarron, R.L. Harlow, and E.H. Moudden, Science 250, 1390 (1990).

7. J.P. Franck, S. Gygax, S. Soerensen, E. Altshuler, A. Hnatiw, J. Jang, M.A.-K. Mohamed, M.K. Yu, G.I. Sproule, J. Chrzanowski, and J.C. Irwin, Physica C185-189, 1379 (1991).

8. G.M. Zhao, M.B. Hunt, H. Keller, and K.A. Müller, Nature 385, 236 (1997).
9. J. Hofer, K. Conder, T. Sasagawa, G.M. Zhao, M. Willemin, H. Keller, and K. Kishio, Phys. Rev. Lett. 84, 4192 (2000).

10. R. Khasanov, D.G. Eshchenko, H. Luetkens, E. Morenzoni, T. Prokscha, A. Suter, N. Garifianov, M. Mali, J. Roos, K. Conder, and H. Keller, Phys. Rev. Lett. 92, 057602 (2004).

11. C. Grimaldi, E. Cappelluti, and L. Pietronero, Europhys. Lett. 42, 667 (1998).

12. A.S. Alexandrov, Europhys. Lett. 56, 92 (2001).

13. T. Schneider and H. Keller, Phys. Rev. Lett. 86, 4899 (2001)

14. A. Deppeler and A.J. Millis, Phys. Rev. B65, 224301 (2002).

15. P. Paci, M. Capone, E. Cappelluti, S. Ciuchi, C. Grimaldi, and L. Pietronero, Phys. Rev. Lett. 94, 036406 (2005).

16. A. Lanzara, P.V. Bogdanov, X.J. Zhou, S.A. Kellar, D.L. Feng, E.D. Lu, T. Yoshida, H. Eisaki, A. Fujimori, J.-I. Shimoyama, T. Noda, S. Uchida, Z. Hussain, and Z.-X. Shen, Nature 412, 510 (2001).

17. G.-H. Gweon, T. Sasagawa, S.Y. Zhou, J. Graf, H. Takagi, D.-H. Lee, and A. Lanzara, Nature 430, 187 (2004).

18. A.F. Hebard, M.J. Rosseinsky, R.C. Haddon, D.W. Murphy, S.H. Glarum, T.T.M. Palstra, A.P. Ramirez, and A.R. Kortan, Nature 350, 600 (1991).

19. K. Tanigaki, T.W. Ebbesen, S. Saito, J. Mizuki, J.S, Tsai, Y. Kubo, and S. Kuroshima, Nature 352, 222 (1991).

20. R.M. Fleming, A.P. Ramirez, M.J. Rosseinsky, D.W Murphy, R.C. Haddon, S.M. Zahurak, and A.V. Makhija, Nature 352, 787 (1991).

21. K. Holczer, O. Klein, S.-M. Huang, R.B. Kaner, K.-J. $\mathrm{Fu}$, R.L. Whetten, and F. Diederich, Science 252, 1154 (1991)

22. J. Nagamatsu, N. Nakagawa, T. Muranaka, Y. Zenitani, and J. Akimitsu, Nature 410, 549 (2001).

23. Y.J. Uemura, L.P. Le, G.M. Luke, B.J. Sternlieb, W.D. Wu, J.H. Brewer, T.M. Riseman, C.L. Seaman, M.B. Maple, M. Ishitawa, D.G. Hinks, J.D. Jorgensen, G. Saito, and H. Yamochi, Phys. Rev. Lett. 66, 2665 (1991).

24. Y.J. Uemura, A. Keren, L.P. Le, G.M. Luke, B.J Sternlieb, W.D. Wu, J.H. Brewer, R.L. Whetten, S.M. Huang, S. Lin, R.B. Kaner, F. Diederich, S. Donovan, G. Grüner, and K. Holczer, Nature 352, 605 (1991).

25. A.B. Migdal, Zh. Eksp. Teor. Fiz. 34, 1438 (1958) [Sov. Phys. JETP 7, 996 (1958)].

26. L. Pietronero and S. Strässler, Europhys. Lett. 18, 627 (1992)

27. G. Grimvall, The Electron-Phonon Interaction in Metals, North-Holland, Amsterdam (1981).

28. A.A. Abrikosov, L.P. Gorkov, and I.E. Dzyaloshinski, Methods of Quantum Field Theory in Statistical Physics, Dover, New York (1975).

29. G. Rickayzen, Green's Functions and Condensed Matter, Academic Press, London (1980). 
30. G.M. Eliashberg, Zh. Eksp. Teor. Fiz. 38, 966 (1960) [Sov. Phys. JETP 11, 696 (1960)].

31. L. Pietronero, Europhys. Lett. 17, 365 (1992).

32. I.G. Lang and Yu.A. Firsov, Zh. Eksp. Teor. Fiz. 42, 1843 (1962) [Sov. Phys. JETP 16, 1301 (1963)].

33. A.S. Alexandrov, Phys. Rev. B38, (1988).

34. A.S. Alexandrov and N.F. Mott, Polarons and Bipolarons, World Scientific, Singapore (1995).

35. A.J. Millis, R. Mueller, and B.I. Shraiman, Phys. Rev. B54, 5389 (1996).

36. S. Ciuchi, F. de Pasquale, S. Fratini, and D. Feinberg, Phys. Rev. B56, 4494 (1997).

37. L. Pietronero, S. Strässler, and C. Grimaldi, Phys. Rev. B52, 10516 (1995).

38. C. Grimaldi, L. Pietronero, and S. Strässler, Phys. Rev. B52, 10530 (1995).

39. C. Grimaldi, L. Pietronero, and S. Strässler, Phys. Rev. Lett. 75, 1158 (1995).

40. A.S. Alexandrov and P.P. Edwards, Physica C331, 97 (2000).

41. V.N. Kostur and P.B. Allen, Phys. Rev. B56, 3105 (1997).

42. A.S. Alexandrov, Europhys. Lett. 56, 92 (2001).

43. M. Grabowski and L.J. Sham, Phys. Rev. B29, 6132 (1984).

44. V.N. Kostur and B. Mitrovic, Phys. Rev. B48, 16388 (1993).

45. V.N. Kostur and B. Mitrovic, Phys. Rev. B50, 12774 (1994)

46. E.J. Nicol and J.K. Freericks, Physica C235, 2379 (1994).

47. J.K. Freericks, V. Zlatic, W.K. Chung, and M. Jarrell, Phys. Rev. B58, 11613 (1998).

48. P. Miller, J.K. Freericks, and E.J. Nicol, Phys. Rev. B58, 14498 (1998).

49. H.R. Krishnamurthy, D.M. Newns, P.C. Pattnaik, C.C. Tsuei, and C.C. Chi, Phys Rev. B49, 3520 (1994).

50. P. Paci, E. Cappelluti, C. Grimaldi, and L. Pietronero, Phys. Rev. B65, 012512 (2002).

51. C. Grimaldi, L. Pietronero, and M. Scattoni, Eur. Phys. J. B10, 247 (1999).

52. M. Grilli and C. Castellani, Phys. Rev. B50, 16880 (1994).

53. M.L. Kulic and R. Zeyher, Phys. Rev. B49, 4395 (1994).

54. R. Zeyher and M.L. Kulic, Phys. Rev. B53, 2850 (1996).

55. M.L. Kulic, Phys. Rep. 338, 1 (2000).

56. Z.B. Huang, W. Hanke, E. Arrigoni, and D. J. Scalapino, Phys. Rev. B68, 220507 (2003).

57. E. Cappelluti, B. Cerruti, and L. Pietronero, Phys. Rev. B69, 161101 (2004).

58. G. Baym and L.P. Kadanoff, Phys. Rev. 124, 287 (1961).

59. L.P. Kadanoff and G. Baym, Quantum Statistical Mechanics, Benjamin, New York (1962).

60. D.J. Scalapino, in: Superconductivity, D.R. Parks (ed.), Dekker, New York (1969).

61. J.P. Carbotte, Rev. Mod. Phys. 62, 1027 (1990).
62. M. Scattoni, C. Grimaldi, and L. Pietronero, Europhys. Lett. 47, 588 (1999).

63. E. Cappelluti, C. Grimaldi, and L. Pietronero, Phys. Rev. B64, 125104 (2001).

64. M. Botti, E. Cappelluti, C. Grimaldi, and L. Pietronero, Phys. Rev. B66, 054532 (2002).

65. P. Benedetti, C. Grimaldi, L. Pietronero, and G. Varelogiannis, Europhys. Lett. 28, 351 (1994).

66. P.W. Anderson, J. Phys. Chem. Solids 11, 26 (1959).

67. D. Fay and J. Appel, Phys. Rev. B20, 3705 (1979).

68. D. Fay and J. Appel, Phys. Rev. B22, 1461 (1980).

69. C. Grimaldi and L. Pietronero, Europhys. Lett. 47, 681 (1999).

70. S. Engelsberg and J.R. Schrieffer, Phys. Rev. 131, 993 (1963).

71. F. Marsiglio, M. Schossmann, and J.P. Carbotte, Phys. Rev. B37, 4965 (1988).

72. E. Cappelluti and L. Pietronero, Phys. Rev. B68, 224511 (2003).

73. X.J. Zhou, T. Yoshida, A. Lanzara, P.V. Bogdanov, S.A. Kellar, K.M. Shen, W.L. Yang, F. Ronning, T. Sasagawa, T. Kakeshita, T. Noda, H. Eisaki, S. Uchida, C.T. Lin, F. Zhou, J.W. Xiong, W.X. Ti, Z.X. Zhao, A. Fujimori, Z. Hussain, and Z.-X. Shen, Nature 423, 398 (2003).

74. O. Gunnarsson, Rev. Mod. Phys. 69, 575 (1997).

75. W.E. Pickett, in: Solid State Physics, v. 48, H. Ehrenreich and F. Spaepen (eds.), Academic Press, New York (1994).

76. E. Cappelluti and L. Pietronero, Phys. Status Solidi B242, 133 (2005).

77. For a review see: R.S. Markiewicz, J. Phys. Chem. Solids 58, 1179 (1997).

78. E. Dagotto, Rev. Mod. Phys. 66, 763 (1994).

79. P. Fulde, Electron Correlations in Molecules and Solids, Springer Verlag, Heidelberg (1995).

80. Correlated Electron Systems, V.J. Emery (ed.), World Scientific, Singapore (1993).

81. A. Georges, G. Kotliar, W. Krauth, and M. Rozenberg, Rev. Mod. Phys. 88, 13 (1996).

82. M.C. Gutzwiller, Phys. Rev. Lett. 10, 159 (1963).

83. M. Lavagna, Phys. Rev. B41, 142 (1990)

84. J.H. Kim and Z. Tesanovic, Phys. Rev. Lett. 71, 4218 (1993).

85. J.D. Lee, K. Kang, and B.I. Min, Phys. Rev. B51, 3830 (1995).

86. M. Mierzejewski, J. Zielinski, and P. Entel, Phys. Rev. B57, 590 (1998).

87. T. Yildirim, L. Barbedette, J.E. Fischer, C.L. Lin, J. Robert, P. Petit, and T.T.M. Palstra, Phys. Rev. Lett. 77, 167 (1996).

88. J. Bernholc, Phys. Today 52, 30 (1999).

89. C.M. Varma, J. Zaanen, and K. Raghavachari, Science 254, 989 (1991).

90. M. Schluter, M. Lannoo, M. Needels, G.A. Baraff, and D. Tománek, Phys. Rev. Lett. 68, 526 (1992).

91. M. Schluter, M. Lannoo, M. Needels, G.A. Baraff, and D. Tománek, J. Phys. Chem. Solids 53, 1473 (1992). 
92. O. Gunnarsson and G. Zwicknagl, Phys. Rev. Lett. 69, 957 (1992).

93. E. Koch, O. Gunnarsson, and R.M. Martin, Phys. Rev. Lett. 83, 620 (1999).

94. J.E. Han, O. Gunnarsson, and V.H. Crespi, Phys. Rev. Lett. 90, 167006 (2003).

95. E. Cappelluti, C. Grimaldi, L. Pietronero, and S. Strässler, Phys. Rev. Lett. 85, 4771 (2000).

96. E. Cappelluti, C. Grimaldi, L. Pietronero, S. Strässler, and G.A. Ummarino, Eur. Phys. J. B21, 383 (2001).

97. J.C. R. Faulhaber, D.Y.K. Ko, and O.R. Briddon, Phys. Rev. B48, 661 (1993).

98. V.P. Antropov, O. Gunnarsson, and A.I. Liechtenstein, Phys. Rev. B48, 7651 (1993).

99. S.K. Watson, K. Allen, D.W. Denlinger, and F. Hellman, Phys. Rev. B55, 3866 (1997).

100. M.S. Fuhrer, K. Cherrey, A. Zettl, M.L. Cohen, and V.H. Crespi, Phys. Rev. Lett. 83, 404 (1999).

101. For a review see: Y. Iwasa and T. Takenobu, J. Phys.: Condens. Matter 15, 495 (2003) and references therein.

102. O. Zhou, R.M. Fleming, D.W. Murphy, M.J. Rosseinsky, A.P. Ramirez, R.B. van Dover, and R.C. Haddon, Nature 362, 433 (1993).

103. M.R. Norman, D. Pines, and C. Kallin, cond-mat/0507031 (2005).

104. J. Rossat-Mignod, L.P. Regnault, C. Vettier, P. Bourges, P. Burlet, J.Y. Henry, and G. Lapertot, Physica C185-189, 86 (1991).

105. V. Barzykin and D. Pines, Phys. Rev. B52, 13585 (1995).

106. P. Monthoux and D. Pines, Phys. Rev. B50, 16015 (1994).

107. Ch. Renner, B. Revaz, J.-Y. Genoud, K. Kadowaki, and O. Fischer, Phys. Rev. Lett. 80, 149 (1998).

108. V.M. Loktev, R.M. Quick, and S.G. Sharapov, Phys, Rep. 349, 1 (2001).

109. M. Grilli, R. Raimondi, C. Castellani, C. Di Castro, and G. Kotliar, Phys. Rev. Lett. 67, 259 (1991).

110. J.M. Tranquada, J.D. Axe, N. Ichiwara, A.R. Moodenbaugh, Y. Nakamura, and S. Uchida, Phys. Rev. Lett. 78, 338 (1997)

111. V.J. Emery and S.A. Kivelson, Physica C209, 597 (1993).

112. V.J. Emery and S.A. Kivelson, Physica C235, 189 (1994).

113. C. Castellani, C. Di Castro, and M. Grilli, Z. Phys. B103, 137 (1997).

114. R.S. Markiewicz, J. Phys. Chem. Solids 59, 1737 (1998).

115. J.W. Loram, K.A. MIrza, J.R. Cooper, and J.L. Tallon, J. Phys. Chem. Solids 10-12, 2091 (1998).

116. E. Cappelluti and R. Zeyher, Phys. Rev. B59, 6475 (1999).

117. S. Onoda and M. Imada, J. Phys. Soc. Jpn. Suppl. B69, 32 (2000).

118. J. Stajic, A. Iyengar, K. Levin, B.R. Boyce, and T. Lemberger, Phys. Rev. B68, 024520 (2003).
119. L. Boeri, E. Cappelluti, C. Grimaldi, and L. Pietronero, Phys. Rev. B68, 214514 (2003).

120. C.C. Tsuei, J.R. Kirtley, C.C. Chi, L.S. Yu-Jahnes, A. Gupta, T. Shaw, J.Z. Sun, and M.B. Ketchen, Phys. Rev. Lett. 73, 593 (1994).

121. G. Blumberg, A. Koitzsch, A. Gozar, B.S. Dennis, C.A. Kendziora, P. Fournier, and R.L. Greene, Phys. Rev. Lett. 88, 107002 (2002).

122. J.A. Skinta, M.-S. Kim, T.R. Lemberger, T. Greibe, and M. Naito, Phys. Rev. Lett. 88, 207005 (2002).

123. A. Biswas, P. Fournier, M.M. Qazilbash, V.N. Smolyaninova, H. Balci, and R.L. Greene, Phys. Rev. Lett. 88, 207004 (2002).

124. N.-C. Yeh, C.-T. Chen, G. Hammerl, J. Mannhart, A. Schmehl, C.W. Schneider, R.R. Schulz, S. Tajima, K. Yoshida, D. Garrigus, and M. Strasik, Phys. Rev. Lett. 87, 087003 (2001).

125. Y. Dagan and G. Deutscher, Phys. Rev. Lett. 87, 177004 (2001)

126. A. Sharoni, O. Millo, A. Kohen, Y. Dagan, R. Beck, G. Deutscher, and G. Koren, Phys. Rev. B65, 134526 (2002)

127. A.I. Lichtenstein and M.L. Kulic, Physica C245, 186 (1995)

128. P. Paci, C. Grimaldi, and L. Pietronero, Eur. Phys. J. B17, 235 (2000).

129. See for instance: P.B. Allen, Z. Fisk, and A. Migliori, in: Physical Properties of High-Temperature Superconductors, D.M. Ginsberg (ed.), World Scientific, Singapore (1989)

130. E. Cappelluti and L. Pietronero, Europhys. Lett. 36, 619 (1996).

131. E. Cappelluti and L. Pietronero, Phys. Rev. B53, 932 (1996).

132. J. Kortus, I.I. Mazin, K.D. Belashchenko, V.P. Antropov, and L.L. Boyer, Phys. Rev. Lett. 86, 4656 (2001)

133. J.M. An and W.E. Pickett, Phys. Rev. Lett. 86, 4366 (2001).

134. T. Yildirim, O. Gülseren, J.W. Lynn, C.M. Brown, T.J. Udovic, Q. Huang, N. Rogado, K.A. Regan, M.A. Hayward, J.S. Slusky, T. He, M.K. Haas, P. Khalifah, K. Inumaru, and R.J. Cava, Phys. Rev. Lett. 87, 037001 (2001).

135. Y. Kong, O.V. Dolgov, O. Jepsen, and O.K. Andersen, Phys. Rev. B64, 020501 (2001).

136. A.Y. Liu, I.I. Mazin, and J. Kortus, Phys. Rev. Lett. 87, 087005 (2001).

137. K.-P. Bohnen, R. Heid, and B. Renker, Phys. Rev. Lett. 86, 5771 (2001).

138. E. Cappelluti, S. Ciuchi, C. Grimaldi, L. Pietronero, and S. Strässler, Phys. Rev. Lett. 88, 117003 (2002).

139. E. Cappelluti, S. Ciuchi, C. Grimaldi, and L. Pietronero, Phys. Rev. B68, 174509 (2003).

140. L. Boeri, G.B. Bachelet, E. Cappelluti, and L. Pietronero, Phys. Rev. B65, 214501 (2002).

141. L. Boeri, E. Cappelluti, and L. Pietronero, Phys. Rev. B71, 012501 (2005). 